\title{
Separating Gravitational Wave Signals from Instrument Artifacts
}

\author{
Tyson B. Littenberg \\ Maryland Center for Fundamental Physics, Department of Physics, \\ University of Maryland, College Park, MD 20742 and \\ Gravitational Astrophysics Laboratory, NASA Goddard Spaceflight Center, 8800 Greenbelt Rd., Greenbelt, MD $207 r 1$
}

Neil J. Cornish

Department of Physics, Montana State University, Bozeman, MT 59717

(Dated: August 11, 2010)

\begin{abstract}
Central to the gravitational wave detection problem is the challenge of separating features in the data produced by astrophysical sources from features produced by the detector. Matched filtering provides an optimal solution for Gaussian noise, but in practice, transient noise excursions or "glitches" complicate the analysis. Detector diagnostics and coincidence tests can be used to veto many glitches which may otherwise be misinterpreted as gravitational wave signals. The glitches that remain can lead to long tails in the matched filter search statistics and drive up the detection threshold. Here we describe a Bayesian approach that incorporates a more realistic model for the instrument noise allowing for fluctuating noise levels that vary independently across frequency bands, and deterministic "glitch fitting" using wavelets as "glitch templates", the number of which is determined by a trans-dimensional Markov chain Monte Carlo algorithm. We demonstrate the method's effectiveness on simulated data containing low amplitude gravitational wave signals from inspiraling binary black hole systems, and simulated non-stationary and non-Gaussian noise comprised of a Gaussian component with the standard LIGO/Virgo spectrum, and injected glitches of various amplitude, prevalence, and variety. Glitch fitting allows us to detect significantly weaker signals than standard techniques.
\end{abstract}

\section{INTRODUCTION}

To take full advantage of gravitational wave detectors we must confidently distinguish weak gravitational wave $(\mathrm{GW})$ signals from detector noise. This challenge, dubbed the "detection problem," warrants significant attention as we prepare for the first direct measurement of gravitational waves within the next decade. In their current operational configuration, the Laser Interferometer Gravitational wave Observatory (LIGO) [1] and Virgo 2], encounter frequent large-amplitude transient noise events, or "glitches", while detectable GW signals are forecast to be rare and weak. The LIGO-Virgo collaboration has developed several practical techniques for addressing the detection problem in this context (see e.g. Refs. 3, [4]), but there is considerable room for improvement.

We propose a new approach to the detection problem that works by simultaneously modeling the instrument noise and the gravitational wave signals. It can be argued that the existing search algorithms incorporate implicit noise modeling as the search statistics are tuned using time slides of the data (the time shifts destroy the coherence of the gravitational wave signals while preserving the noise properties in a statistical sense). In our Bayesian approach to the detection problem we must use an $e x$ plicit noise model, which in turn defines the likelihood function. We wish to stress that the issue is not whether a Bayesian or frequentist approach is superior, but rather that the "tuning" of the search must proceed in a different fashion. In the frequentist approach, one or more statistics are chosen that (hopefully) indicate whether a gravitational wave signal is present in the data. Time slides and signal injections are then used to produce estimates of the false alarm and false dismissal probabilities for the statistics. The choice of statistics can be refined during this procedure in an effort to minimize the false dismissals and false alarms. In a Bayesian approach the analysis is fully determined by the choice of likelihood function and prior. Once these have been chosen the analysis is purely mechanical, there are no thresholds to set or statistics to tune. As Laplace put it "the theory of probabilities is basically just common sense reduced to calculus" [5]. Though the well defined probability calculus of Bayesian analysis is appealing, the output is only as good as the input. Bayesian analyses of LIGO-Virgo data that assume a Gaussian likelihood function [6 6 should be treated with caution.

Rather than making guesses about the noise model, and hence the form of the likelihood function, Bayesian inference can be used to determine the noise model from the data [9]. To this end, we introduce several parameterized models for the noise, and use the data to jointly estimate the noise and signal parameters. Bayesian model selection is applied to alternative parameterizations of the noise in an effort to find the most parsimonious representation. The process of designing these parameterized likelihood models is similar to the process of designing statistics for a frequentist analysis. The main difference is that the "tuning" of the likelihood models occurs mechanically, without fear of operator induced biases.

We consider a variety of noise models, and in tests performed on simulated LIGO/Virgo noise, we find that a model that combines a description of Gaussian noise with 
a time varying power spectrum and a wavelet model that is able to fit semi-coherent glitches best represents the data. While we were careful to ensure that the models used to generate the simulated data did not correspond exactly to the models being tested, it is certainly no surprise that the model favored by the analysis is the one that is most similar to the model used to simulate the data. Indeed, the main motivation for working with simulated data is to have full control of the experiment - if the analysis had not selected the likelihood model that is closest to the injected noise model we would know that something was wrong. The next step, which we are actively pursuing, is to repeat our analysis using real LIGO/Virgo data.

We are not alone in promoting the idea that better noise modeling is key to the gravitational wave detection problem. Allen et al. [9, 10] have argued that parameterized noise models are needed to derive robust search statistics. Clark et al. [11] included a model for SineGaussian instrument glitches as an alternative hypothesis when computing Bayesian odds ratios for gravitational wave signals from pulsar glitches. Clark et al. also suggested that it would be valuable to have a classification of instrument glitches that could be used to construct better models for the instrument noise. Similar "glitch hypotheses" were considered by Veitch and Vecchio [12] in a study of Bayesian model selection applied to the search for black hole inspiral signals. The possibility of subtracting instrument glitches from the data using a physical model of the detector has been investigated by Ajith et al. [13, 14]. Principe and Pinto 15] have introduced a physically motivated model for the glitch contribution to the instrument noise, and have used this model to derive what they refer to as a "locally optimum network detection statistic" [16]. In their approach the glitches are treated in a statistical sense, while our approach directly models the glitches present in the data. The possibility of directly deriving likelihood functions from the data in a Bayesian setting has previously been considered by Cannon [17]. In Cannon's approach the data is first reduced to an n-tuple of quantities, such as the parameters produced by a matched filter search, then using time slides and signal injections the likelihood distributions for the signal and noise hypotheses are directly estimated from the data. These are then used to estimate the posterior probability that a measured set of parameters corresponds to a gravitational wave event.

We develop our approach as follows: In section ஹwe discuss the connection between noise models and likelihood functions, and in section [II we describe the wavelet representation that we use to define our noise models. Section IV provides a brief review of the Markov Chain Monte Carlo algorithm that we use to carry out the calculus of Bayesian inference. In section $\nabla$ we introduce our noise models and describe the simulated data sets. Bayesian model selection is then applied to identify the most effective noise model. Section VI defines the black hole inspiral waveforms that we inject, and section VII describes the search phase of our analysis. Results are presented in section VIII describing a simultaneous characterization of gravitational wave signals and instrument noise for two simulated data sets. We close with a discussion of our plans for future work in section [X]

\section{LIKELIHOOD MODELS}

For Gaussian noise there is a well known optimal solution to the detection problem based on Wiener matched filtering [18], which can be described in terms of a detection statistic $\rho$ - the matched filter signal-to-noise ratio, or equivalently, the $\log$ likelihood $\log \mathcal{L}=-\chi^{2} / 2$. Suppose the data $s$ produced by a gravitational wave detector were the superposition of instrument noise $n$ and the detector's response to a passing gravitational wave $h^{s}$. Then the signal-to-noise for a template $h$ is defined:

$$
\rho(h)=\frac{(s \mid h)}{(h \mid h)^{1 / 2}},
$$

and the chi-squared residual by

$$
\chi^{2}(h)=(s-h \mid s-h) .
$$

The expectation value of $\rho$ is maximized, and $\chi^{2}$ is minimized, when the template matches the signal, $h=h^{s}$. Here we have used the standard noise weighted inner product for Gaussian noise with one-sided noise spectral density $S_{n}(f)$ :

$$
(a \mid b)=2 \int \frac{a b^{*}+a^{*} b}{S_{n}(f)} d f .
$$

The gravitational wave detection problem is usually described in the frequentist language of false alarms and false dismissals based on Monte Carlo studies of a suitably chosen detection statistic. According to the Neyman-Pearson theorem, the quantity $\rho$ provides an optimal statistic for stationary, Gaussian noise as it yields the maximum detection probability for a given false alarm probability. While there are no similar proofs of optimality for the $\rho$ statistic when applied to the nonstationary and non-Gaussian noise encountered in the LIGO/Virgo detectors, the matched filter SNR or closely related quantities are adopted as search statistics that are then calibrated using Monte Carlo studies of signal injections and scrambled data. Sections of data that are identified as being corrupted by excessive noise or transient instrumental artifacts (glitches) are vetoed prior to the tuning of the search statistic. The development of these vetoes is a complex topic, but the basic idea is to either look for correlations between glitches in the gravitational wave channel and the thousands of diagnostic channels, or to look for statistical patterns. A more detailed description of the vetoing procedures and data quality assessment can be found in Ref. [4].

An alternative to tuning a statistic chosen for its performance with Gaussian noise is to look for new statistics 
that can be shown to be near optimal for relevant forms of non-Gaussian noise [9, 10]. This approach is similar in spirit to what we are proposing.

It is interesting to contrast the frequentist and Bayesian approaches to the detection problem. In the Bayesian approach we simply compute the posterior distribution function $p(h \mid s, \mathcal{M})$ for the waveforms $h$ of model hypothesis $\mathcal{M}$, and the associated model evidence $p(s \mid \mathcal{M})$. These are related by Bayes' theorem to the likelihood $p(s \mid h, \mathcal{M})$ and the prior $p(h \mid \mathcal{M})$ by

$$
p(h \mid s, \mathcal{M})=\frac{p(h \mid \mathcal{M}) p(s \mid h, \mathcal{M})}{p(s \mid \mathcal{M})},
$$

and

$$
p(s \mid \mathcal{M})=\int p(h \mid \mathcal{M}) p(s \mid h, \mathcal{M}) d h
$$

Everything we want to know about the waveform model $\mathcal{M}$ is contained in the posterior distribution, while the evidence allows comparisons to be made between alternative models (e.g. is a GW signal present or not). It is important to emphasize that the Bayesian approach is entirely mechanical, there are no statistics to tune or thresholds to set - you simply go ahead and calculate confidence intervals for the waveform parameters and odds ratios for competing hypotheses. On the other hand, the output of this mechanical process is only as good as the inputs - if the waveform model or the likelihood function is flawed, then the conclusion will also be flawed. We will assume that the waveform model is accurate (see Ref. [19] for a discussion of the biases introduced by inaccurate waveform models), and concentrate our attention on the likelihood function.

The data collected by one or more gravitational wave detectors can be represented by a discrete collection of samples $s_{i}$. These may represent the strain sampled at certain times, Fourier amplitudes, wavelet amplitudes etc. In the absence of a gravitational wave signal, $s_{i}=n_{i}$, and we are looking at samples drawn from the instrument noise distribution $p_{N}\left(n_{i}\right)$. When a signal $h_{i}$ is present we have $n_{i}=s_{i}-h_{i}$, and assuming that the noise and the signal are uncorrelated, the likelihood of observing $s_{i}$ given the waveform $h_{i}$ is simply [20]

$$
p\left(s_{i} \mid h_{i}, \mathcal{M}\right)=p_{N}\left(s_{i}-h_{i}\right),
$$

while the likelihood of observing the full data set $\left\{s_{1}, s_{2}, \ldots, s_{N}\right\}$ is the joint probability

$$
p(s \mid h, \mathcal{M})=p_{N}\left(s_{1}-h_{1}, s_{2}-h_{2}, \ldots, s_{N}-h_{N}\right) .
$$

If the noise in each sample is independent, then the joint distribution is the product of the individual distributions:

$$
p(s \mid h, \mathcal{M})=\prod_{i=1}^{N} p_{N}\left(s_{i}-h_{i}\right) .
$$

The key point is that the likelihood function is nothing other than the probability distribution that describes the instrument noise. And therein lies the problem: The description of the likelihood function, and hence the veracity of the Bayesian approach, is only as good as our understanding of the instrument noise. A solution to this problem is to introduce a model for the noise [9], and to use the data to jointly estimate the noise and signal parameters. We have taken this approach to a limited extent in previous analyses where we treated the noise as Gaussian, but with its variance to be determined from the data 21 23. Here we extend the scope of the noise modeling to consider non-Gaussian tails and inter-sample noise correlations of the type caused by semi-coherent "glitches".

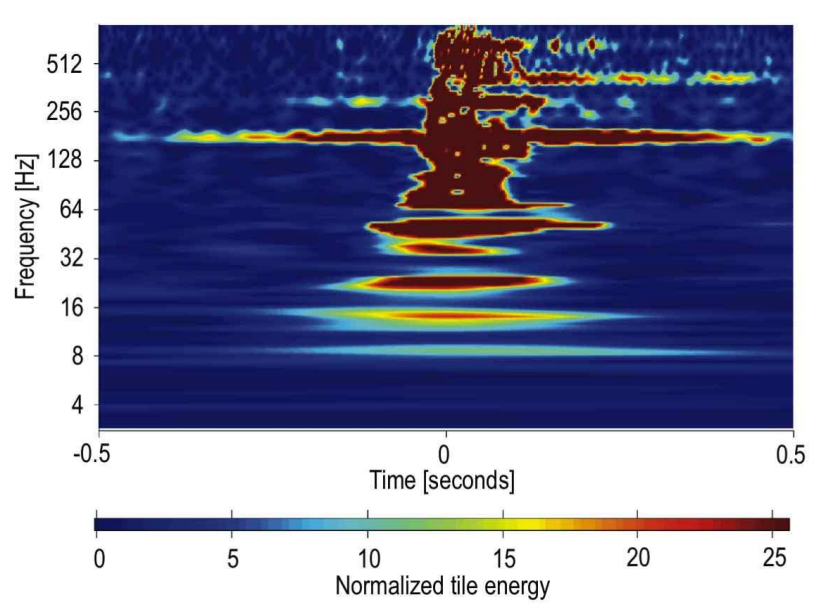

FIG. 1: A time-frequency scaleogram showing a loud glitch in the output of one of the LIGO detectors (LIGO document number LIGO-G070807-00-Z).

Recall that for white Gaussian noise the samples $n_{i}$ will be independent in time and in frequency, while for colored Gaussian noise the samples will be independent in frequency and correlated in time. To avoid the complications of having to account for these correlations with a joint probability distribution, it is standard practice to compute the likelihood in the frequency domain, where the likelihood factors into a simple product [20]. However, as is clear from Figure [1, the glitches seen in the LIGO/Virgo data are semi-coherent in time and frequency - that is, if there is excess noise in one time frequency pixel, then there is a high probability that there will be excess noise in a neighboring time-frequency pixel. These glitches not only produce large non-Gaussian tails, they also introduce strong correlations in the noise model. While there are many ways to try to model this behavior, we will show that treating the glitches as coherent instrumental artifacts provides an effective solution. 


\section{DATA ANALYSIS IN THE WAVELET DOMAIN}

The first step towards constructing a realistic noise model is to abandon the familiar Fourier-domain approach to signal processing in favor of a time-frequency decomposition in the wavelet domain.

Time-frequency methods are employed in a variety of GW search algorithms for LIGO/Virgo data, particularly in searches for gravitational wave "bursts" - transient gravitational wave signals for which no templates are available - such as the coherent Wave Burst (cWB) algorithm [24].

Individual wavelet functions $\psi_{i j}$ are compact in both frequency and time, and can be used to form an orthogonal basis [25]. Each element of the basis is a scaled, time-shifted version of the "mother wavelet" $\psi$ of which a large variety exist. We have chosen the Meyer wavelet to form our basis functions as is used in cWB. Fig. 2 depicts an example of the Meyer wavelet in the time domain, as well as its power spectrum. The Fourier power of a single wavelet function is perhaps most illuminating. Each wavelet basis function acts as a bandpass filter, selecting for a specific range of frequencies, during a specific time interval of the function being decomposed.

We use the discrete wavelet transform (DWT) of a time series $s(t)$ to determine the wavelet coefficients $w_{i j}$. Each $w_{i j}$ represents the amplitude of a pixel in the timefrequency plane with volume $\Delta t \Delta f=1 / 2$. The indices $i$ and $j$ correspond to the frequency and time, respectively, of the wavelet coefficient.

Unique to the DWT is the way the pixels "tile" the time-frequency plane: Low frequency wavelets have long durations and narrow frequency response. As we move to higher frequency the bandwidth of the wavelet increases while the duration shortens (maintaining $\Delta f \Delta t=1 / 2$ ). A cartoon representation of this time-frequency tiling can be found in Fig. 3. Each frequency layer, denoted by the index $i$, contains $2^{i}$ divisions in time and, for signal duration of $T$, is $2^{i-1} / T$ wide in frequency.

For stationary, Gaussian noise, the wavelet coefficients are sufficiently uncorrelated to treat the wavelet-domain noise-correlation matrix as diagonal. In this instance the expectation value of the noise power for the wavelet pixel with indices $i$ and $j, S_{i j}$, can be computed by filtering the noise power spectral density $S_{n}(f)$ with $P_{w}^{i j}(f)=$ $\left|\tilde{\psi}_{i j}(f)\right|^{2}$ :

$$
S_{i j}=\int_{0}^{\infty} P_{w}^{i j}(f) S_{n}(f) d f
$$

The power spectrum for any wavelet in layer $i$, as well as the power spectral density at times $j$ are the same for stationary, Gaussian noise. Including the time index will prove useful when we allow for noise varying with time.

We are now able to define a diagonal noise-weighted inner product as

$$
(a \mid b) \equiv \sum_{k} \sum_{i, j} \frac{a_{i j}^{k} b_{i j}^{k}}{S_{i j}^{k}}
$$

where the index $k$ represents an individual interferometer, or IFO, in the network. For Gaussian noise, the inner products computed in the wavelet domain using (10) are almost identical to the the inner products computed in the Fourier domain using (3).

\section{MARKOV CHAIN MONTE CARLO}

Bayesian methods have lagged behind their frequentist brethren because of the burden associated with evaluating Eqs. (4) and (5). Recently, powerful techniques for overcoming these computational hurdles (e.g., Markov Chain Monte Carlo (MCMC) methods [26, 27], Nested Sampling [28], etc.) have become progressively more efficient and computer power has increased, allowing us to employ these resources on interesting GW detection problems [29, 30].

Our method of choice for computing the posterior distribution function are the MCMC algorithms. These techniques have shown prowess in solving parameter estimation problems, while variants of these methods have also proven effective as search algorithms [31 38]. Here we briefly describe the basis of the algorithms, and leave specific details of our implementation to later sections.

MCMCs provide samples from the (previously unknown) target posterior distribution function for the parameters of some model $\mathcal{M}$. This is accomplished by first adopting a position in parameter space $\vec{\theta}_{x}$ as the first "link" in the chain and evaluating that position's likelihood $p\left(s \mid \vec{\theta}_{x}, \mathcal{M}\right)$ and prior probability $p\left(\vec{\theta}_{x} \mid \mathcal{M}\right)$. Next, we suggest a trial position, $\vec{\theta}_{y}$, from the proposal distribution $q\left(\vec{\theta}_{y} \mid \vec{\theta}_{x}\right)$ read "the probability of suggesting a move to $\vec{\theta}_{y}$ given that the current location is $\vec{\theta}_{x}$ ". The new likelihood and prior probability are evaluated and $\vec{\theta}_{y}$ is adopted as the next link in the chain with probability $\kappa=\min [1, H]$ where $H$ is the Hastings ratio

$$
H_{\vec{\theta}_{x} \rightarrow \vec{\theta}_{y}}=\frac{p\left(s \mid \vec{\theta}_{y}, \mathcal{M}\right) p\left(\vec{\theta}_{y} \mid \mathcal{M}\right) q\left(\vec{\theta}_{x} \mid \vec{\theta}_{y}\right)}{p\left(s \mid \vec{\theta}_{x}, \mathcal{M}\right) p\left(\vec{\theta}_{x} \mid \mathcal{M}\right) q\left(\vec{\theta}_{y} \mid \vec{\theta}_{x}\right)}
$$

This process of stochastically stepping through parameter space repeats until some convergence criteria are satisfied (See Ref. 23] for a description of the convergence tests we use). Afterwards, the number of iterations spent in a particular region of parameter space, normalized by the total number of steps in the chain, yields the probability that the model parameters have values within that region.

The Hastings ratio is derived by mandating transitions from $\vec{\theta}_{x}$ to $\vec{\theta}_{y}$ satisfy the detailed balance condition which 


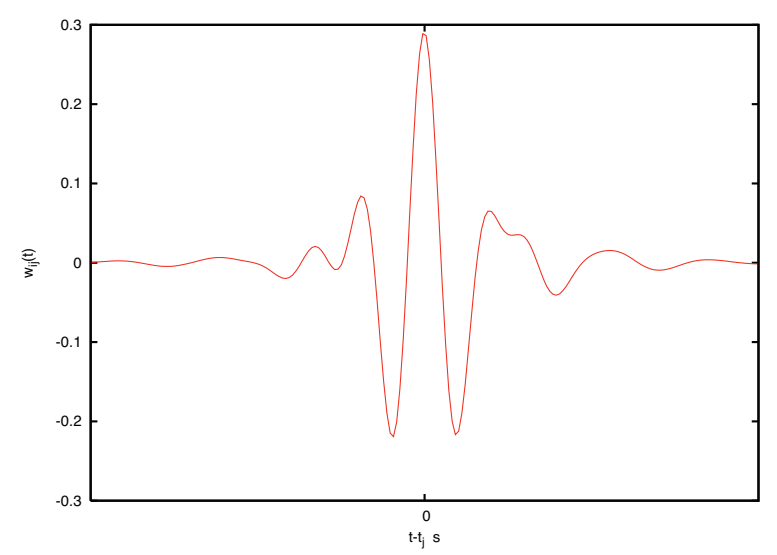

(a) time

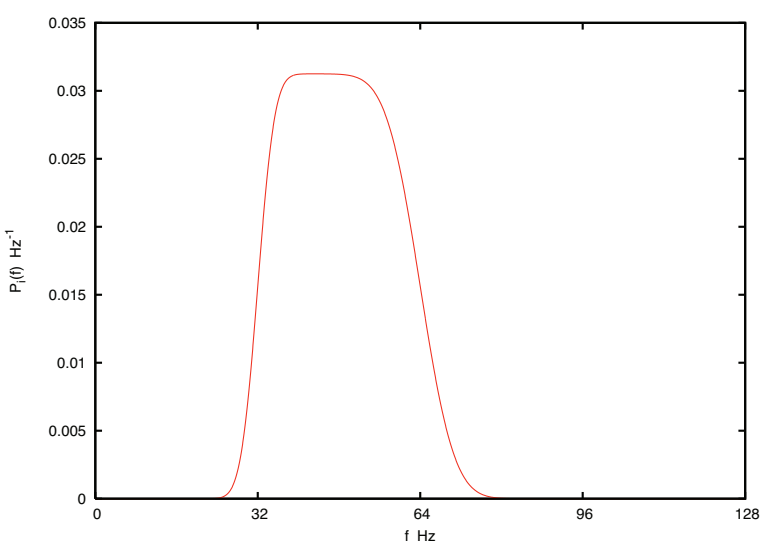

(b) frequency

FIG. 2: The Meyer wavelet basis function for frequency layer $i=9$ and signal duration of 16 seconds. a) Time domain $\psi_{i j}(t)$ for arbitrary time index $j$. b) Fourier power spectrum of $\tilde{\psi}_{i j}(f)$. Notice how, for $i=9$ and $T=16 \mathrm{~s}$, this wavelet acts as a bandpass filter for frequencies $f \sim[32,64] \mathrm{Hz}$.

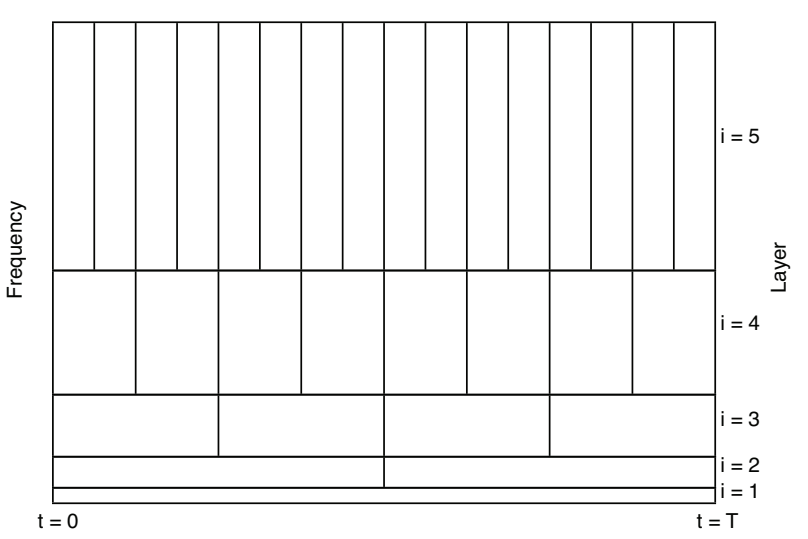

FIG. 3: A cartoon depicting the tiling of the time-frequency plane by a discrete wavelet transform

(DWT). Each pixel has time-frequency volume

$$
\Delta t \Delta f=1 / 2 \text {. }
$$

ensures the samples from the Markov chain are representative of the target PDF.

The choice of $q\left(\vec{\theta}_{y} \mid \vec{\theta}_{x}\right)$, by construction, can not alter the recovered posterior distribution function. The proposal distribution does, however, dramatically affect the acceptance rate of trial locations in parameter space and, therefore, the number of iterations required to satisfactorily sample the joint PDF.

Markov chains are still prone to being "trapped" by local maxima of the target distribution for longer than the user would be willing to wait. Some enhancement to the above prescription is often mandatory in order to ensure global sampling of the PDF. One such method, parallel tempering [39], uses a set of chains running simultaneously, each at a higher "temperature." The likelihood of a chain with inverse temperature $\beta=1 / T$ is calculated by $p(s \mid \vec{\theta}, \mathcal{M})^{\beta}$. Increasing the temperature effectively smoothes the topography of the distribution being explored. High temperature (low $\beta$ ) suppresses the differences in likelihood between the current and proposed model parameters, allowing the high temperature chains free exploration of the parameter space. In the limit where the temperature goes to infinity ( $\beta$ goes to zero) the chain will sample the prior distributions.

Parallel tempering promotes adequate mixing by allowing the chains of different temperature to exchange parameters, thus allowing solutions found by the hot chains to be communicated to the colder chains. This sharing of solutions can be executed while maintaining detailed balance if exchanges are accepted using

$$
H_{i \leftrightarrow j}=\frac{p\left(s \mid \vec{\theta}_{i}, \mathcal{M}_{i}, \beta_{j}\right) p\left(s \mid \vec{\theta}_{j}, \mathcal{M}_{j}, \beta_{i}\right)}{p\left(s \mid \vec{\theta}_{i}, \mathcal{M}_{i}, \beta_{i}\right) p\left(s \mid \vec{\theta}_{j}, \mathcal{M}_{j}, \beta_{j}\right)}
$$

as the Hasting's ratio in the transition probability for an exchange between chain $i$ and $j$. Only points in the $\beta=1$ chain sample the target distribution and are therefore permitted to contribute to the chain from which the PDF is inferred. However, by exchanging parameters with hotter chains the $\beta=1$ samples rapidly explore the full target distribution, including movement between different modes of the posterior which, for practical applications, can be impossible for a single chain to achieve.

While only the $\beta=1$ chain contributes to the PDF, the 
rest of the chains serve as more than just a convergence aid. The average log-likelihood of each chain, integrated over $\beta=[0,1]$, is equivalent to the integral in (5) [40], allowing us to perform parameter estimation and model selection studies using a single analysis "pipeline."

It should be noted that simultaneously solving the parameter estimation and model selection problem is not a unique attribute of the parallel tempered Markov chain Monte Carlo (PTMCMC) algorithm. On the contrary, the MultiNest algorithm [41, 42] also serves as an "all-inone" Bayesian analysis package that has been successfully employed in GW data analysis problems. While we will exclusively use the PTMCMC approach in this paper we want to emphasize that these algorithms are merely tools used to mechanically perform the calculation of (4) and (5), and while some tools may be better suited for a particular problem than others, the conclusions drawn from the data should be identical.

\section{MODELING THE NOISE}

We now return to our goal of finding the best noise model (equivalently the best likelihood function) to be used when describing realistic GW data. We will construct three different likelihood functions and compare their performance on simulated non-Gaussian noise using Bayesian model selection. What follows is a brief summary of each noise model before a more detailed description and comparison:

- Model $N_{0}$ : Allow the noise level to vary as a function of both time and frequency.

- Model $N_{1}$ : Use a likelihood function derived from a distribution which has non-Gaussian tails.

- Model $G_{1}$ : In conjunction with $N_{0}$ or $N_{1}$, model the noise as two independent contributions - a stochastic component drawn from $N_{i}$ plus coherent "glitches."

\section{A. $N_{0}$ : Fitting to the noise level}

The first order solution is to fit to the underlying "DC" noise level in the data. This has been done in frequency domain analyses 21 23] allowing the model enough flexibility to account for errors in the predicted $S_{n}(f)$. In the wavelet basis this approach to noise modeling gains sensitivity to non-stationarities because temporal information is encoded in the noise "spectrum."

For this model, parameterized by $\vec{\eta}$, we use the expected noise level in "blocks" of wavelet pixels containing a certain time-frequency volume (TFV) normalized by the theoretical noise level,

$$
\eta_{i j}^{k}=\frac{S_{i j, \text { measured }}^{k}}{S_{i j, \text { theoretical }}^{k}}
$$

with a single value of $\eta$ assigned to each TFV block:

$$
\eta_{i j_{0}}^{k}=\eta_{i, j_{0}+1}^{k}=\ldots=\eta_{i, j_{0}+\mathrm{TFV}-1}^{k},
$$

where $j_{0}$ is the time index of the first wavelet coefficient in the block under consideration. Because the noise level is allowed to vary, the normalization of the likelihood and the noise weighted inner product in the chi-squared depend on the noise parameters:

$$
\ln p\left(s \mid \vec{\eta}, N_{0}\right)=-\frac{1}{2}\left((r \mid r)+\sum_{k}^{\mathrm{IFO}} \sum_{i, j} \ln \eta_{i j}^{k}\right)
$$

with $\eta_{i j}^{k} S_{i j}^{k}$ substituted for $S_{i j}^{k}$ in the inner product defined by (10). The second term in (15) comes from the normalization of the likelihood. Note that for a noiseonly model, the residual $r$ is simply the data $s$.

If we fit to the noise level in large time-frequency blocks we lose sensitivity to short-duration non-stationarities, while using small time-frequency blocks results in a model with a large number of parameters, all of which must be constrained. In a model selection sense, the former will produce poor fits to the data (if nonstationarities are present) while the latter will carry a large "Occam Factor" penalty for the model's additional degrees of freedom. Some tuning is required to choose the correct TFV for the noise blocks in order to optimize this approach. While $N_{0}$ is a non-stationary noise model, it is unable to respond to short duration impulses of noise unless it is equipped with an unreasonable number of free parameters.

\section{B. $N_{1}$ : Non-Gaussian Tails}

This noise model, denoted by $N_{1}$, will continue to fit to the noise level in blocks of wavelet pixels as in $N_{0}$, while additionally employing the suggestion in Ref. [9] to redefine the likelihood as a weighted sum of two normal distributions. The majority of the weight will be allocated to the distribution with the same variance as a Gaussian model so that the bulk of noise samples are presumably "drawn from" the standard picture of the instrument noise. However, some non-zero contribution to the likelihood comes from a significantly wider distribution. This broadens the tails of the noise distribution without greatly altering it's core. In other words, the majority of samples drawn from this distribution will "look" as though they come from the ideal noise distribution, however the frequency of "outliers" is greatly increased.

Symbolically, the probability of measuring $n_{i}$ in a single bin of the data can be calculated as

$$
p\left(n_{i}\right)=\frac{1}{\sqrt{2 \pi}}\left(\frac{\alpha}{\sigma_{1}} e^{-n_{i}^{2} / 2 \sigma_{1}^{2}}+\frac{1-\alpha}{\sigma_{2}} e^{-n_{i}^{2} / 2 \sigma_{2}^{2}}\right) .
$$

To demonstrate the effect of this distribution, suppose we choose $\sigma_{2}=3 \sigma_{1}$ and $\alpha=0.99$. Then, for a single 
noise sample,

$$
\frac{p\left(n=0 \mid N_{1}\right)}{p\left(n=0 \mid N_{0}\right)}=0.993
$$

and

$$
\frac{p\left(n=10 \sigma_{1} \mid N_{1}\right)}{p\left(n=10 \sigma_{1} \mid N_{0}\right)}=1.970 \times 10^{18} .
$$

We see that for samples near the mean, the difference between the two distributions is negligible, while there is a large increase in the probability of large noise excursions under $N_{1}$.

Following the discussion deriving Eq. (8), the likelihood of measuring the residual $r$ in the wavelet domain for $N_{1}$ becomes

$$
p\left(s \mid \vec{\eta}, N_{1}\right)=\prod_{k} \prod_{i j} p\left(r_{i j}^{k}\right) .
$$

Using a superposition of two normal distributions is a standard method in Bayesian modeling of non-gaussian data [43] but has not previously been implemented in gravitational wave signal processing.

\section{C. $G_{1}$ : Fitting for Glitches}

While both $N_{0}$ and $N_{1}$ possess many of the qualities which we desire in an accurate noise model, both assume that each noise sample in the data is independent (in the wavelet domain). Instrument glitches, on the other hand, are coherent in time-frequency space. A wavelet scaleogram representation, depicted in Figure 1 is a useful visualization of this feature, as the glitch can be seen "lighting up" a large cluster of pixels, while the remaining data samples are well described as being independently, randomly distributed.

We require some way of modeling the individual glitches, and a template bank of such events would undoubtedly be inefficient and incomplete. Instead we can once more make use of the wavelet basis, and model the glitches as linear combinations of the basis functions. Studies of LIGO/Virgo glitches using atoms (e.g., sineGaussians) have shown that typical events can be decomposed with $\sim 10$ basis functions [15, 16] so it is reasonable to anticipate a similarly small number of wavelets will be sufficient. The parameters for the glitch fitting are the number of wavelets included in the fit, $n_{G}$, the indices identifying where in time-frequency space those wavelets (referred to as "hot pixels") are located, and their amplitudes $\vec{\gamma}$.

It is unknown a priori how many, or where in timefrequency space, these hot pixels will be needed. Given this uncertainty we must automatically adjust the number and location of wavelet coefficients in the glitch fitting, a task best accomplished by the Reversible Jump Markov Chain Monte Carlo (RJMCMC) 44] approach.
This breed of MCMC is able to transition between models of differing dimension while satisfying detailed balance if the probability of a trans-dimensional move is computed using

$$
H_{\mathcal{M}_{i} \rightarrow \mathcal{M}_{j}}=\frac{p\left(s \mid \vec{\theta}_{j}, \mathcal{M}_{j}\right) p\left(\vec{\theta}_{j}, \mathcal{M}_{j}\right) q\left(\vec{\theta}_{i}, \mathcal{M}_{i}\right)}{p\left(s \mid \vec{\theta}_{i}, \mathcal{M}_{i}\right) p\left(\vec{\theta}_{i}, \mathcal{M}_{i}\right) q\left(\vec{\theta}_{j}, \mathcal{M}_{j}\right)}\left|J_{i j}\right|
$$

as the Hasting's ratio in the transition probability. The Jacobian $\left|J_{i j}\right|$ accounts for any change in dimension between models $\mathcal{M}_{i}$ and $\mathcal{M}_{j}$. The number of samples spent in each model, normalized by the total number of samples in the chain, is the posterior probability, or evidence, for that model (assuming adequate mixing, convergence, etc.).

In our glitch-fitting RJMCMC, trans-dimensional moves propose to either remove a single pixel from the glitch model (setting a wavelet coefficient to zero), or include a new pixel which was previously not a part of the glitch model (selecting a wavelet coefficient which is zero, and assigning it some amplitude).

Because the number and location of hot pixels is variable, model $G_{1}$ is more aptly described as a "metamodel," while a particular number and configuration of hot pixels represents a discrete model for the noise. We can denote an individual glitch model by it's number of hot pixels, $n_{G}$. For model $n_{G}$ and data containing $N$ samples, there are " $N$ choose $n_{G}$ " $=K_{n}$ unique combinations of hot pixels. We want each configuration to have equal prior probability, so the joint prior probability for each noise model configuration is

$$
p\left(n_{G} \mid G_{1}\right)=1 / K_{n}=\frac{n_{G} !\left(N-n_{G}\right) !}{N !} .
$$

Meanwhile, we choose as the prior on the pixel amplitudes $p\left(\gamma_{i j} \mid n_{G}, G_{1}\right)=N\left[0,100 S_{i j}\right]$.

RJMCMCs are often difficult to work with because of inefficient mixing between dimensions. The key to overcoming this obstruction is to construct trans-dimensional proposal distributions which closely match the target distribution. We do so by taking advantage of a glitch's tendency to excite a cluster of pixels in time-frequency space. The trans-dimensional proposal distributions thus favor the inclusion of new pixels in the glitch model which are adjacent to the already existing clusters. We do so by assigning each unoccupied pixel a probability of being selected for inclusion in the glitch model which is weighted by the number of neighboring pixels already "lit up." Meanwhile, the trans-dimensional proposal distribution for the glitch amplitudes is identical to that of the prior on the amplitudes, thus canceling in the Hasting's ratio.

\section{Simulating Non-Gaussian Noise}

To test our approaches to noise modeling, before applying these techniques to data collected by the LIGOVirgo network, we will analyze simulated data containing 
Gaussian noise with injected glitches. We use a variety of glitch injections to ensure that any success is not a result of tuning the methods to a particular noise realization. We begin by generating Fourier-domain noise for a 16 second data segment using the initial LIGO/Virgo design sensitivities. We can inject glitches of two different type into the data. One which we will refer to as the "gaussian-gaussian" type, is a burst of gaussian noise enveloped by a gaussian profile in time. The second are the glitch atoms described in Ref. [15].

Glitches are either placed in the data deliberately, or randomly chosen such that the glitches are distributed uniformly in the time-frequency plane, with average durations of 10 "cycles." Each generated glitch $g_{0}$, is rescaled to achieve a desired "SNR" $=\sqrt{(g \mid g)}$. The glitch SNR, $x$, is either chosen by hand, or drawn from a double-power law distribution

$$
p(x)=C\left(x^{-4}+x_{i}^{-5 / 2} x^{-3 / 2}\right), \quad x>x_{0}
$$

calibrated to the Black Hole ring-down trigger rate in a segment of "typical" LIGO S5 data. $C$ is a normalization constant and $x_{i} \sim 10$. The total number of injections was also determined by the ring-down trigger rates. This distribution produces glitches which are predominantly buried by the Gaussian component of the noise, with only a rare $(\sim 1$ in 5$)$ noise realization containing an event visually above the Gaussian noise. By injecting glitches from this distribution we are ruining the stationarity and Gaussianity of the noise without using our models for the noise to produce the samples.

\section{E. Comparison of Noise Models}

To compare the relative performance of our new approaches to noise modeling, we analyzed three different signal realizations, $s_{1}$ : Gaussian noise; $s_{2}$ : Gaussian noise plus a single, loud (SNR $\sim 100$ ) glitch; and $s_{3}$ : Gaussian noise plus 300 glitches distributed across the network with SNRs drawn from (22). We use the same Gaussian noise realization in each simulation.

Each data set was analyzed using the four different combinations of noise models described above: $\left[N_{0}, G_{0}\right]$, $\left[N_{1}, G_{0}\right],\left[N_{0}, G_{1}\right]$, and $\left[N_{1}, G_{1}\right]$, for noise blocks of different time frequency volume (TFV). Histograms showing the evidence for each model as a function of $\log _{2}(\mathrm{TFV})$, as well as the posterior for the hot-pixel number $n_{G}$ for $s_{3}$, are displayed in Figure 4.

We will first address the dependence of the model evidence on TFV. For $s_{1}$ and $s_{3}$ the data were dominated by Gaussian noise, while $s_{2}$ contained a significantly "loud", short duration, glitch. Intuitively, noise models with fewer degrees of freedom are preferable when the noise level from block to block hardly strays from the theoretical prediction $\left(s_{1}\right.$, and to some extent, $\left.s_{3}\right)$, while models with more flexibility should be favored when a large deviation from Gaussian noise exists. This presumption is supported by the results as we see the evidence improves with increasing TFV (decreasing number of noise parameters) for $s_{1}$ and $s_{3}$, and diminishes (considering models without glitch fitting $\left.\left(G_{0}\right)\right)$ for $s_{2}$.

This study has also allowed us to make some conclusions about selecting between these different models for non-Gaussian noise. We anticipated that the favored model would most qualitatively resemble the noise simulation, to wit, $\left[N_{0}, G_{0}\right]$ for $s_{1},\left[N_{0}, G_{1}\right]$ for $s_{2}$, and $\left[N_{1}\right.$, $\left.G_{0}\right]$ for $s_{3}$. This prediction, however, is not entirely supported by the results.

For $s_{1}$ we see that $\left[N_{0}, G_{0}\right]$ is on either equal footing (for small TFV), or mildly disfavored (at large TFV), when competing with $\left[N_{1}, G_{0}\right]$ and $\left[N_{0}, G_{1}\right]$. While $\left[N_{0}, G_{0}\right]$ is the noise model which most faithfully represents how the noise for $s_{1}$ was generated, it's inflexibility when compared to $\left[N_{0}, G_{1}\right]$ and $\left[N_{1}, G_{0}\right]$ makes it less well suited to cope with a particular noise realization. It should be pointed out, however, that the differences in evidence in this example are not significant in a modelselection sense.

For $s_{2}$ we see a strong preference for models which include glitch fitting $\left(G_{1}\right)$. This is to be expected when the data contain a large amplitude noise impulse, while the $G_{0}$ models are more adept at describing instrument noise with more frequent, lower amplitude, and uncorrelated noise excursions.

The data simulation which is arguably the most relevant to our ambition of including this work in the analysis of current gravitational wave data would be $s_{3}$. Here we have a large number of glitches (300) injected into the data with SNRs drawn from a distribution calibrated to match the frequency of black hole ring-down triggers in S5 data. Our motivation for this realization is to produce a signal that is most like the actual interferometer data. The resulting noise "looks" predominantly Gaussian, as the vast majority of the glitches are well below the normal instrument noise level. However, when the data are analyzed with the noise models we see a generic disfavoring of the Gaussian model $\left[N_{0}, G_{0}\right]$. Furthermore, we are pleasantly surprised to see that $\left[N_{0}, G_{1}\right]$ performs as well as $\left[N_{1}, G_{0}\right]$ in this example even though $\left[N_{1}, G_{0}\right]$ is qualitatively best suited for this noise simulation.

The final point of interest regarding the performance of the different noise models is the generally underwhelming performance of $\left[N_{1}, G_{1}\right]$. The expectation going in to the study was that it would outperform its counterparts on $s_{3}$, as it was best suited to handle both the rare impulsive events, and the overall non-Gaussian component from the superposition of many small-amplitude glitches. To explain why it generally underperforms when compared to the other two non-Gaussian models we refer to Fig. 4. The plot shows the posterior distribution functions for the number of hot pixels $n_{G}$ used by the glitch fitting. The Gaussian likelihood model, $\left[N_{0}, G_{1}\right]$ (red), has a peak in the posterior at $n_{G}=4$, while $\left[N_{1}, G_{1}\right]$ 's peak (blue) is closer to zero, at $n_{G}=1$. If the noise model uses a Gaussian distribution for the likelihood some number of 


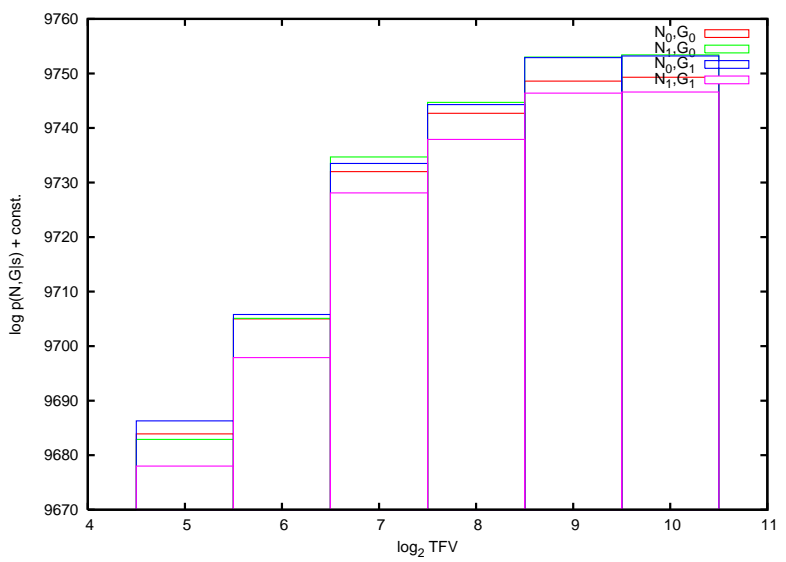

(a) $s_{1}$

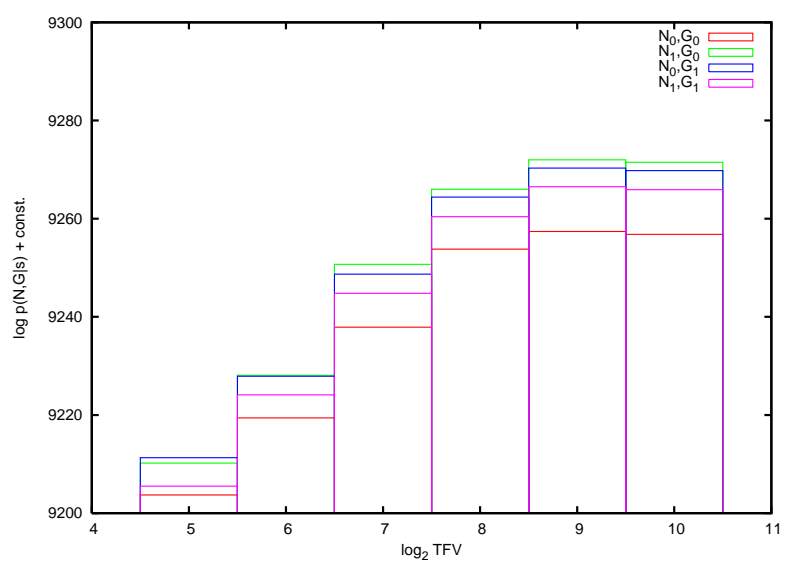

(c) $s_{3}$

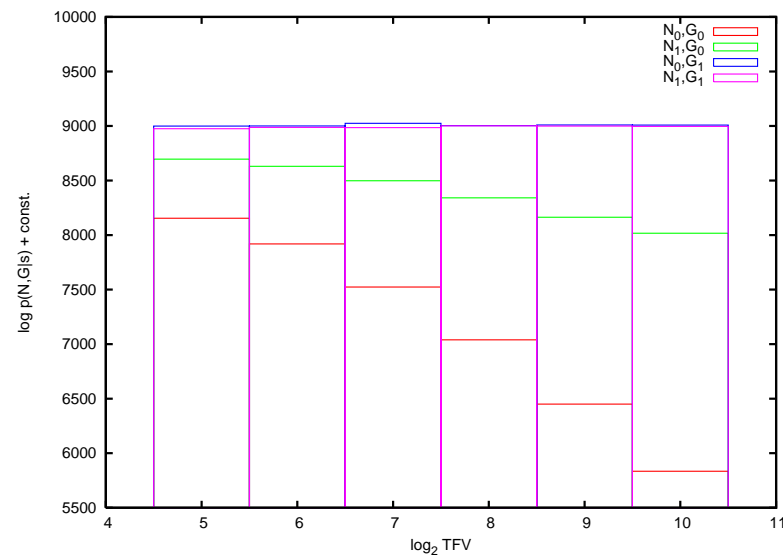

(b) $s_{2}$

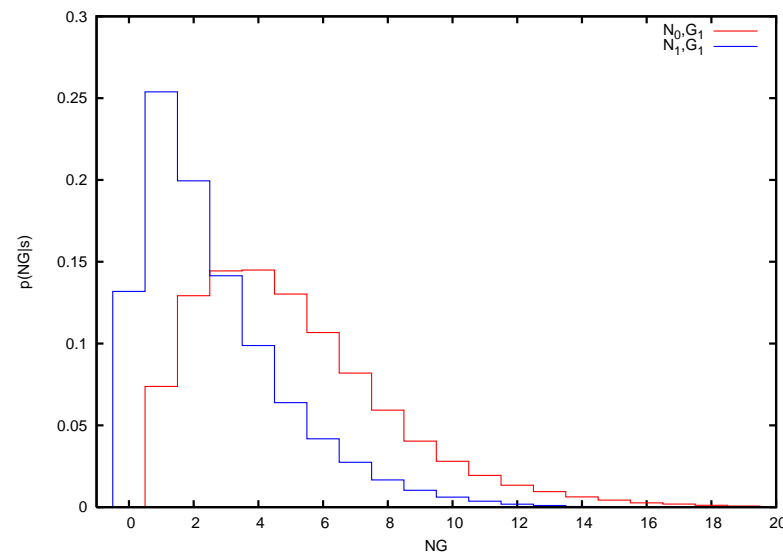

(d) $p\left(m \mid N_{i}, G_{1}\right)$ for $s_{3}$

FIG. 4: Plots (a)-(c) show the log evidence for different noise models applied to signal realizations $\left(s_{1}, s_{2}, s_{3}\right)$ as a function of the TFV used by $N_{0}$ and $N_{1}$. Models with discernibly higher evidence are preferred. Plot (d) shows the distribution of the "hot-pixel" number used by $G_{1}$ for the signal realization $s_{3}$. Our interpretation of these results is discussed in the text.

hot pixels are required to achieve a sufficiently Gaussian residual, and $G_{1}$ has the flexibility to do so. However, if the likelihood accounts for these non-Gaussian excursions by having more weight at large $\sigma$, fewer hot pixels are needed. $N_{1}$ is, by itself, an accurate representation of the noise and adding $G_{1}$ to the modeling introduces additional degrees of freedom without a substantial benefit to the overall fit, making it a less attractive choice in a model selection sense.

Another unanticipated result can be seen in Fig. $4 \mathrm{~d}$. We generically see that the posterior distribution on $n_{G}$ is always peaked away from zero. This can be understood by studying the Hasting's ratio for transitions from $n_{G}=$ 0 to $n_{G}=1$. Under such transitions,

$$
H_{0 \rightarrow 1}=\frac{p(s \mid 1)}{p(s \mid 0)} \frac{p(i, j \mid 1)}{p(i, j \mid 0)} \frac{p\left(\gamma_{i j} \mid 1\right)}{p\left(\gamma_{i j} \mid 0\right)} \frac{q(i, j \mid 0)}{q(i, j \mid 1)} \frac{q\left(\gamma_{i j} \mid 0\right)}{q\left(\gamma_{i j} \mid 1\right)}
$$

with

$$
q(i, j \mid 0)=1: \text { there is only one pixel to remove in }
$$

a transition from $1 \rightarrow 0$.

$$
\begin{aligned}
q(i, j \mid 1)=1 / N & : \text { there are } N \text { equally likely pixels } \\
& \text { to include in a transition } 0 \rightarrow 1 . \\
p(i, j \mid 0)=1: & \text { there is only one configuration of } \\
& \text { pixels if none of them are "hot." } \\
p(i, j \mid 1)=1 / N: & \text { there are } N \text { configurations for } \\
& \text { a single "hot" pixel. }
\end{aligned}
$$

$p\left(\gamma_{i j} \mid m\right)=q\left(\gamma_{i j} \mid m\right)$ : by construction.

and $H_{0 \rightarrow 1}$ reduces to the likelihood ratio. Therefore, as long as the inclusion of a single hot pixel does not increase the residual, $p(s \mid 1)>p(s \mid 0), H_{0 \rightarrow 1}>1$, and the transition to $n_{G}=1$ is accepted.

The results of these noise model comparisons lead us to the following conclusion: While more than one model is preferred under different circumstances (noise/glitch realizations), the model which uses a Gaussian likelihood along with glitch fitting was never seen to be disfavored 
in comparison to the other models under consideration. Given this generic utility, we will adopt $\left[N_{0}, G_{1}\right]$ as our default noise model while turning our attention to the model selection challenge in which we are more fundamentally interested: Distinguishing between a gravitational wave signal and non-Gaussian noise.

\section{MODELING THE GRAVITATIONAL WAVES}

We take as our test signal the inspiral of a binary system composed of two stellar-mass black holes. The full gravitational waveform from the inspiral of binary black holes is composed of an inspiral, merger, and ringdown phase. Analytic solutions exist for the inspiral and ringdown waveforms courtesy of post-Newtonian and black hole perturbation theory, respectively. Recent advances in Numerical Relativity have led to the simulation of black-hole mergers, allowing (for particular combinations of mass and spin) the construction of full waveforms. Analytic models of the full waveforms, calibrated against the numerical simulations, provide accurate waveforms which cover the entire signal (see e.g [46, 47]). While a true analysis pipeline should utilize all of the available signal, for this proof-of-principle effort we will only simulate the inspiral phase of waveform both for our injections as well as our analysis.

While neglecting the post-inspiral part of the waveforms, we apply additional simplifications by ignoring spin effects, as well as any orbital eccentricity. What remains is a model of the waveforms which is characterized by nine quantities written as components of the signal-model parameter vector:

$$
\vec{\lambda} \rightarrow\left(m, \mathcal{M}, t_{c}, \log D_{L}, \sin \delta, \alpha, \cos \theta_{L}, \phi_{L}, \varphi_{c}\right)
$$

where $m$ and $\mathcal{M}$ are the total and chirp mass of the binary system, $t_{c}$ is the time when the binary coalesces (more accurately, when the post-Newtonian approximation to the frequency diverges), $D_{L}$ is the luminosity distance to the system, $\delta$ and $\alpha$ are the declination and right ascension as defined on the celestial sphere, $\theta_{L}$ and $\phi_{L}$ are the polar coordinates of the angular momentum vector for the binary, and $\varphi_{c}$ is the GW phase at coalescence. We will further simplify the waveform by only considering the quadrupole radiation, ignoring higher harmonics. For parameter estimation studies which incorporate the details that we have ignored see, for example, 45, 4850].

\section{THE DETECTION ALGORITHM}

Armed with models for the noise and the signals, we now wish to test their relative distinguishability on simulated data using the PTMCMC detection algorithm. We will consider four models:

$$
\begin{aligned}
& N_{0}, G_{0}, B_{0}: s(t, f)=n(t, f), \\
& N_{0}, G_{0}, B_{1}: s(t, f)=n(t, f)+h(t, f), \\
& N_{0}, G_{1}, B_{0}: s(t, f)=n(t, f)+g(t, f), \\
& N_{0}, G_{1}, B_{1}: s(t, f)=n(t, f)+g(t, f)+h(t, f),
\end{aligned}
$$

and for each, thoroughly resolve the PDF of the model parameters and calculate the evidence. We will consider the prior odds between the models to be unity so that differences in model evidence are equal to differences in the posterior probability for each model. Henceforth, we will omit writing $N_{0}$ as part of the model identification as it is common in all scenario's considered here.

The detection algorithm is broadly divided into three phases. For each model under consideration the algorithm performs a:

- Search: To locate the regions of high probability in parameter space.

- Characterization: To globally sample the posterior distribution for the model parameters.

- Evaluation: To calculate model evidence and determine which (if any) of the models are favored.

The stages of the algorithm are linked automatically, making this an "end-to-end" analysis pipeline for matched-filtering searches. Generally, the search phase sacrifices detailed balance in favor of rapid convergence, and uses a modest number of parallel chains $(\sim 10)$. Information from the search is used to construct informed proposal distributions for the characterization, where we must take care that the chains are Markovian. During the characterization phase we employ a stronger dose of parallel tempering ( $\sim 30$ chains) to thoroughly sample the evidence integrand. When using thermodynamic integration of the evidence, the evaluation phase occurs in post-processing as all of the necessary information is acquired during the characterization phase.

The above description of the detection algorithm is a very general outline of the procedure. What follows is a more detailed look into the implementation of each step for this study.

\section{A. The Glitch Search}

We first analyze the data from each detector individually with the glitch-only model $\left(G_{1}, B_{0}\right)$, to establish the range of hot pixels over which the model posterior has significant support. The noise in each detector is independent, as is the noise model (including glitch fitting). Therefore, we need not perform this phase of the analysis simultaneously across the network.

While the $\left[G_{1}, B_{0}\right]$ chains are running in their postburn-in stage, the state of the glitch model (i.e., the number, location, and amplitude of non-zero wavelet coefficients used in the glitch fitting) is periodically stored 
so that we retain a population of samples from the glitch search chains.

This "glitch cleaning" approach would not work if the data contained un-modeled bursts of gravitational waves as the wavelets efficiently fit the astrophysical signal (as is demonstrated in the wavelet-domain LSC burst search algorithms). We can perform the analysis in stages here because the power in a single bin of data from a black hole binary signal is swamped by the instrument noise, and it is only with matched filtering that we can effectively elevate these signals above the noise. The glitch model is therefore "blind" to the $\mathrm{BH}$ waveform in the data, and is left to clean the noise down to some Gaussian residual. In $\S$ IX we describe an improved approach that combines the glitch fitting in each detector with a search for coherent power across the detector network.

\section{B. The Gravitational Wave Search}

The goal of the GW search phase is to locate the modes of the posterior distribution function. Straight forward implementations of MCMCs converge slowly - impractically so when exploring large spaces of high dimensionality. Several methods exist for optimizing a search using the Metropolis-Hastings algorithm as the driver for the exploration [31 38]. The most effective are those which either violate detailed balance and/or sample from a biased target distribution (e.g. simulated annealing, Fstatistic extremization, "mode-hopping" proposals, etc.). There do exist enhancements which preserve the independence of the chain samples, such as delayed rejection, but we have found the implementation of such methods unnecessarily complicated.

Our approach is to use "illegal" search techniques to locate the regions of high posterior weight and to use the biased PDF from this search as one of the proposal distributions used to sample from the target distribution. Specifically, we maximize the likelihood over a subset of the extrinsic parameters, $\left\{t_{c}, \varphi_{c}, D_{L}\right\}$ using the correlation of the template with the data. This maximization must be done in the Fourier domain and assumes gaussian noise. The search is therefore performed in frequency space and in the absence of noise fitting. Consequently, the search-phase inner products use the initial LIGO and Virgo theoretical noise PSD.

The disadvantage to this Fourier-domain, biased search phase lies in our inability to do any glitch fitting during this stage. To prevent the searching chains from spending time fitting to glitches, we make use of the stored states from the glitch removal phase. A state of the (already finished) glitch chain is randomly chosen, and subtracted from the data. The subtraction is done in the timedomain, and occurs periodically, about once every 1000 iterations, so the search phase sees the residual from different realizations of the glitch-only analysis. Performing this maneuver while maintaining detailed balance would be a challenge but because we are merely localizing the modes of the posterior (and not claiming that we are accurately sampling the PDF), we can get away with these violations.

\section{Characterization and Evaluation}

To characterize the signal and glitch model, and evaluate each model's evidence, we repeat the analysis with the full implementation of parallel tempering and noise fitting while satisfying detailed balance and sampling from the target distribution. We greatly expedite this phase of the analysis by including the proposal distribution that was constructed from the (biased) samples of the GW search phase. Recall that the proposal distribution, by construction of the Hastings ratio, can not bias the chain's sampling of the target distribution. Even though the search-chain samples were obtained "illegally', they can be used to help move the chains between modes of the target distribution.

To form this efficient proposal distribution, we sort the GW search chain parameters into a joint histogram with bin widths (in each parameter direction) equal to some multiple of the standard deviation for that parameter as estimated by the Fisher Information Matrix. The Fisher Information Matrix is evaluated at the search chain's Maximum a Posteriori (MAP) parameters. The technique of constructing a proposal distribution for a highdimensional space which has been sparsely covered by a Markov chain was described with more detail in [23]. Binning the search chains into a proposal distribution can most likely be made more efficient by using binary space partitioning algorithms - a technique that has previously been studied for use in LIGO/Virgo analyses to approximate the posterior distribution from a Markov chain [52].

Provided the resulting 9-dimensional histogram built out of the search-chain samples is normalized and allows access to the entire prior volume it is a perfectly valid proposal distribution from which we can draw new parameter combinations (taking care to accurately calculate the $q(x \mid y) / q(x \mid y)$ term in the Hastings ratio). This proposal distribution, although biased by the likelihood maximization from the search phase, is a sufficiently accurate approximation to the true PDF to improve the mixing of the chains during the characterization phase. The use of parallel tempering, coupled with the pilot exploration of the posterior, leads to rapid (less than $10^{3}$ iterations) convergence and supplies further assurance that the chain is globally sampling the target distribution.

We further expedite matters by restricting the number of hot pixels in the glitch model to those which had significant weight in the search-phase posterior. Effectively, we are reducing the number of glitch models under consideration and neglecting those which would contribute negligible weight to the evidence.

Upon the completion of the characterization chains, the average likelihood for each chain is computed, and the evidence is calculated using a simple trapezoid inte- 
gration over the inverse temperature.

\section{Prior distributions}

We use uninformative (flat) priors on all of the signal model parameters with angular variables taking the full allowed range. We allow for the individual constituents of the binary to have masses ranging from $1 \mathrm{M}_{\odot}$ to $30 \mathrm{M}_{\odot}$. The distance to the binary is constrained between $10^{-1}$ and $10^{3} \mathrm{Mpc}$. Because the masses are scale parameters we use uniform distributions in the log of the chirp and total mass. Each time-frequency block for which a noise parameter is assigned contains $N_{T F V}=1024$ pixels.

For more detail regarding the implementation of the MCMC and parallel tempering, refer to the Appendix.

\section{RESULTS}

The data are simulated by first creating a noise realization as described in $\$ \mathrm{~V}$, and then injecting a black hole waveform with the luminosity distance tuned to achieve the desired network signal-to-noise ratio $\mathrm{SNR}=\sqrt{(h \mid h)}$. The injected SNR is computed using the theoretical, Gaussian noise level, so the actual SNRs will be somewhat lower since additional noise is injected by way of the glitches. We will study the performance of the detection algorithm on two noise simulations qualitatively similar to $s 2$ and $s 3$ from $\$ \mathrm{~V}$ over a three-detector network which includes both four kilometer LIGO interferometers plus Virgo.

\section{A. Example 1: Network of Detectors, Single Loud Glitch}

For this example, the BH injections had SNRs between 6 and 12. The glitch injection contained a single glitch atom, injected into the simulated Virgo data, with a SNR of 100. The time-frequency location of the glitch was chosen to overlap with the $\mathrm{BH}$ waveform.

Figure 5 shows the accuracy with which the glitch is removed from the data. The top panel displays the full 16 seconds of Virgo's time-domain data (red) and the residual (blue) for a typical state of the chain. The lower panel zooms in to the region around the glitch, and includes the injected Gaussian noise (black). The RJMCMC glitch fitting procedure was able to parsimoniously remove the non-Gaussian component of the noise without significantly affecting the rest of the data.

The distributions of the number of hot pixels $\left(n_{G}\right)$ used by the RJMCMC glitch model are shown in Figure 6. The value of $n_{G}$ at the peak of these distributions is the glitch model with the highest evidence. The favored models for the LIGO Livingston and Hanford detectors (LHO and LLO) are for low $n_{G}$ as the simulated noise from those instruments was stationary and Gaussian, while
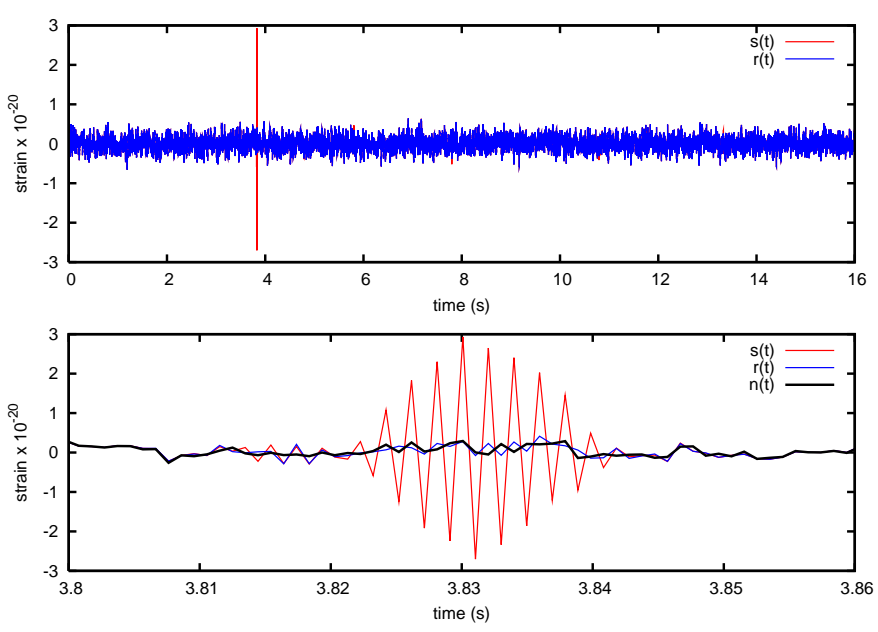

FIG. 5: A typical residual from the glitch search phase. The top panel shows the full 16 seconds of Virgo data while the bottom is a zoomed in view of the glitch. The red line is for the raw data $s(t)$, while the blue line is the residual $r(t)$ from a randomly selected state of the glitch-search chain. Included in the lower panel is the

injected Gaussian noise $n(t)$ demonstrating the accuracy of the glitch removal.

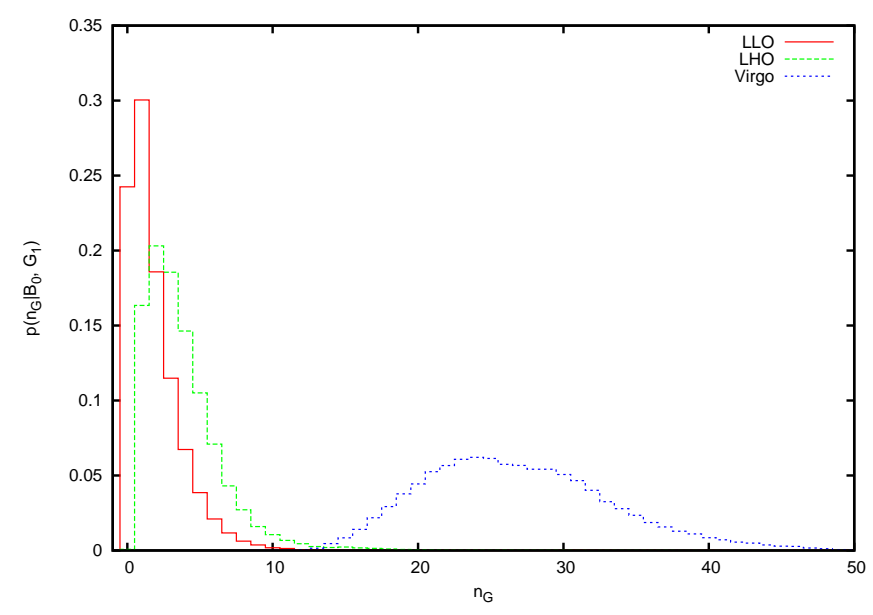

FIG. 6: PDF of $n_{G}$ for stationary, Gaussian noise with a single "loud" glitch injected into the Virgo data. The RJMCMC automatically prefers small values of $n_{G}$ for the Gaussian noise, and $\sim 25$ glitch wavelets to fit the

large amplitude noise excursion seen in Figure 5.

we see clear evidence for a non-Gaussian feature in the Virgo data. For the characterization/evaluation studies we restrict $n_{G}$ in each interferometer to those in Figure 6 which have non-zero probability. For discussion of why the LLO and LHO histograms are not peaked at zero see g.

The black hole search was run on data with the glitch already removed as described in \$VII. For demonstrative purposes, we also ran a search on the "raw" data 


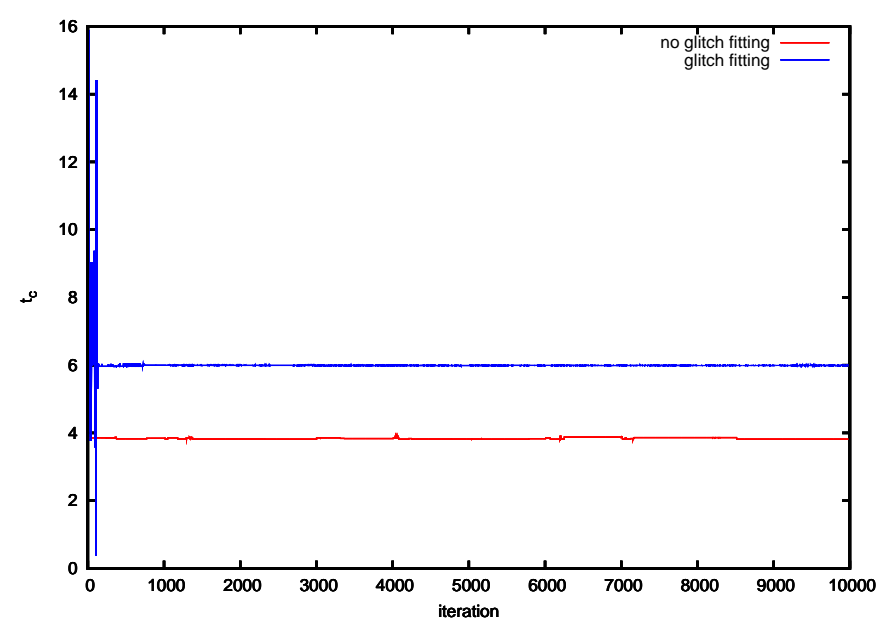

FIG. 7: Time-to-coalescence search chains with (blue) and without (red) glitch fitting for Example 1. Without glitch fitting the search locks onto a glitch in the Virgo detector (injection time $\sim 4 \mathrm{~s}$ ) while with glitch fitting the search locks onto the signal injection $\left(t_{c} \sim 6 \mathrm{~s}\right)$. The

GW signal was injected with SNR of 12 . The prescription for the glitch fitting is described in $\$ \mathrm{VII}$.

still containing the glitches. Figure 7 shows the timeto-coalescence chains for the searches performed on the data with the $\mathrm{BH}$ injection at $\mathrm{SNR}$ of 12 . The search templates on the raw data had $t_{c}$ values consistent with the time of the glitch injection (completely missing the $\mathrm{BH}$ signal), while the chains exploring the residual data found the correct value of the merger time. For the other data sets, the injected source parameters were successfully extracted from the residual data for injections above signal-to-noise ratios of $\sim 8$.

Upon completing the search chains, we bin the samples from the $\mathrm{BH}$ search into a 9D histogram to use as a proposal distribution (as described in \$VII and Ref. 23] ) and reanalyze the data using the four models outlined in the previous section. During this characterization phase the noise and signal parameters are updated together, and care is taken to satisfy the detailed balance condition.

Figure 8 shows the posterior distribution functions for each of the BH signal parameters, marginalized over all other parameters and noise models, for the $\mathrm{SNR}=12$ signal injection. Vertical lines denote the injected parameter values, all of which lie in the supported region of the posterior. The familiar multi-modal structure of these posterior distributions is in evidence.

The evidence for each model is calculated using thermodynamic integration and the results of these calculations are shown in Figure 9. The evidence calculation is un-normalized, so the value for each model is of no consequence. What matters is the relative evidence between the different descriptions of the data. For all cases, the models with glitch fitting $\left(G_{1}\right)$ dominate over those without $\left(G_{0}\right)$. For the $G_{1}$ models, we begin to see significant support for the black-hole model $\left(B_{1}\right)$ at SNR of
10. There is never significant support for the noise-only model because we set the prior range on $D_{L}$ to extend well beyond the range of the detectors, i.e., included in our GW model are signals which are undetectable. The model selection scheme should not distinguish between noise only or noise plus a gravitational wave of (near) zero amplitude. Had we adopted some minimum SNR cutoff on the luminosity distance, we would have seen the noise model being favored at low SNR. A similar study was performed for galactic binaries in Ref. [23].

The question of "detectability" is more clearly demonstrated by looking at the Bayes Factor

$$
\mathcal{B}_{1,0}=\frac{p\left(s \mid G_{1}, B_{1}\right)}{p\left(s \mid G_{1}, B_{0}\right)}
$$

shown in Figure 10. Because of our choice to use uniform priors on the different models, $\mathcal{B}_{1,0}$ is equivalent to the odds ratio for favoring the $\mathrm{BH}$ model to the noise-only model. The dashed lines represent odds ratios of 3:1 and 12:1 which are historically taken as different "confidence" intervals in Bayesian model selection. For Bayes factors between 3:1 and 12:1 model 1 is weakly supported over model 0, while above 12:1 the support for model 1 is considered strong. We find that by $\mathrm{SNR}=10$ there is a clear preference for the signal model which then increases exponentially with the gravitational wave amplitude.

Perhaps more importantly, we do not see false positive detections (i.e., the $\mathrm{BH}$ model fitting to the glitch with significant evidence) when the $\mathrm{BH}$ amplitude is too low to be detected. This contrasts with the models that treat the noise as Gaussian $\left(G_{0}\right)$ where we see strong support for the BH model even though the GW chains never located the injected signal (see Figure 9).

\section{B. Example 2: Network of Detectors, Glitch Distribution}

Example 1 is a simplified simulation of non-Gaussian noise for the ground based GW detectors. To further test the effectiveness of our noise-modeling algorithm we simulated new data and re-ran the analysis described in Example 1 but with 100 glitches injected from a distribution calibrated off of the S5 ring-down trigger rate as described in $8 \mathrm{~V}$. Spectrograms of the glitch injections into each detector are shown in Figure 11 and are compared to the waveform injection which is shown using the same color scale. Figure 12 shows the various contributions to the detector output for the Virgo detector, which happened to have the loudest glitch in this noise realization (at $t \sim 1$ second).

The results for this second example are qualitatively similar to those for the first example, thus the discussion in this section is kept brief. Figures [13, 14, 15] for Example 2 correspond to Figures 7, 9, 10 for Example 1.

Despite the increased complexity of this data simulation, and the increased challenge of extracting the GW 

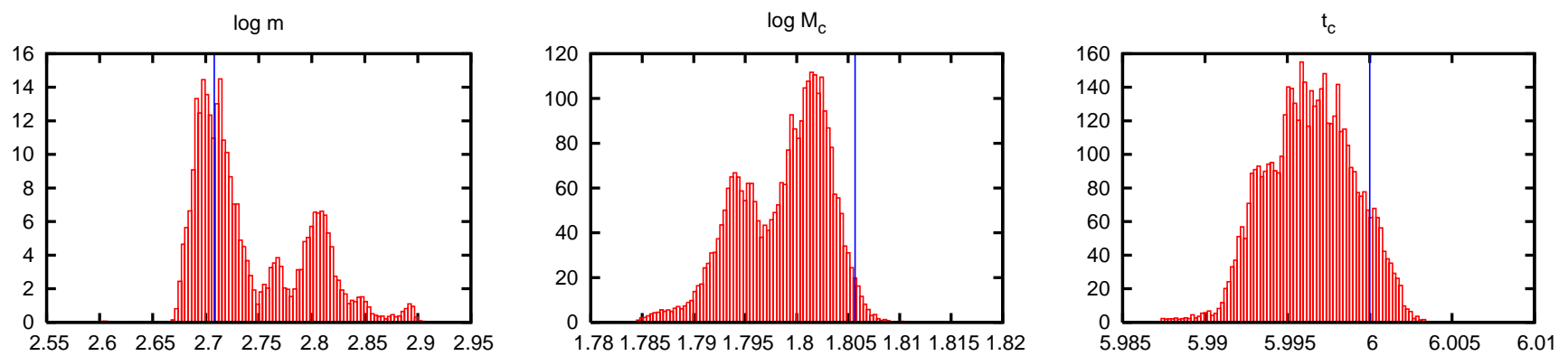

$D_{L}$
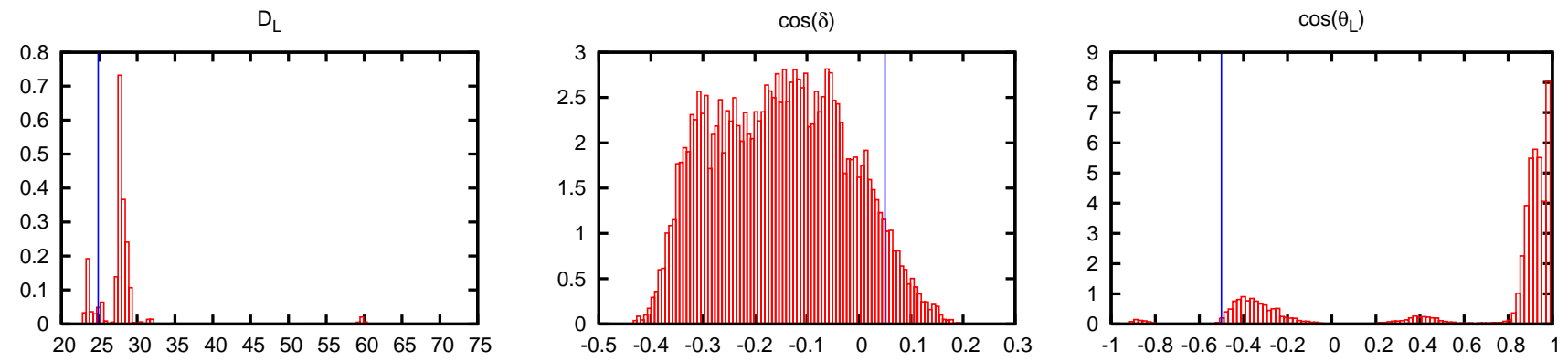

$\phi_{\mathrm{L}}$
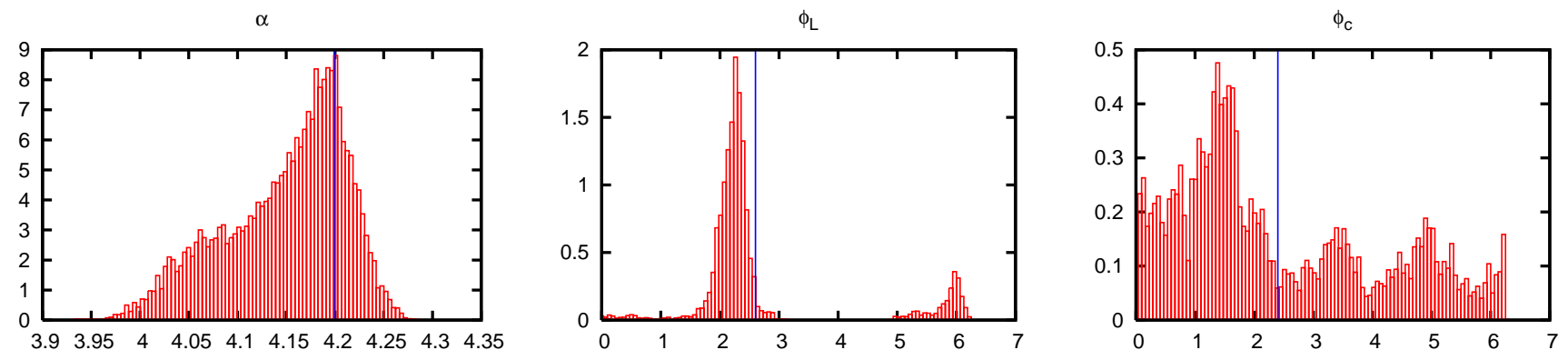

FIG. 8: Marginalized PDFs of $\vec{\lambda}$ for SNR $=12$ from Example 1. The vertical line marks the injected waveform's values. Notice the multi-modal structure of the posteriors, illustrating the challenge these signals pose for any analysis method.

signal from the non-Gaussian noise, the results and conclusion made from this example are essentially identical to those from the simpler "single glitch" case of Example 1. The only significant differences between this and the previous test are the degree to which glitch fitting improves the evidence, and to the overall confidence levels of detection.

The change in evidence between $G_{0}$ and $G_{1}$ models is much larger in Example 1, where the injected glitch was a much more predominant feature in the data. However, the relative difference between evidence with and without glitch fitting, for both examples shown here, demonstrate overwhelming support for $G_{1}$ models.

We attribute the slight differences in the detection confidences to the GW signal injections, which are scaled to achieve a desired ideal SNR $=\sqrt{(h \mid h)}$. Because the data from Example 2 contains, on average, more power in each wavelet layer (due to the additional, irresolvable, lowSNR glitches) as compared to Example 1, the effective SNR of the glitch injections is lower than the indicated by the theoretical SNR that appears along the x-axis.
The evidence ratios for the models that include glitch fitting shown in Figure 15 should be compared to the evidence ratios found with no glitch fitting that are shown in Figure 16. Here the dangers of using a Gaussian likelihood function are thrown into stark relief. A fully convergent Bayesian calculation of the odds ratio favors the signal model even when no signal is injected! It is not until the injected signal has SNR above 16 that the recovered parameters actually correspond to what was injected. In contrast, the glitch fitting model only favors the signal model when the injected signal parameters are recovered. Veitch and Vecchio [51] have also observed that using a Gaussian noise model to calculate Bayes factors on glitchy data can lead to "false positives", and they have argued that this can be accounted for by treating the odds ratio as a frequentist statistic, that can be tuned using signal injections and time slides of the data. While this suggestion is not without merit, it comes at the cost of having much higher detection thresholds than those demonstrated here when the noise model includes glitch fitting. It is worth mentioning that the current 

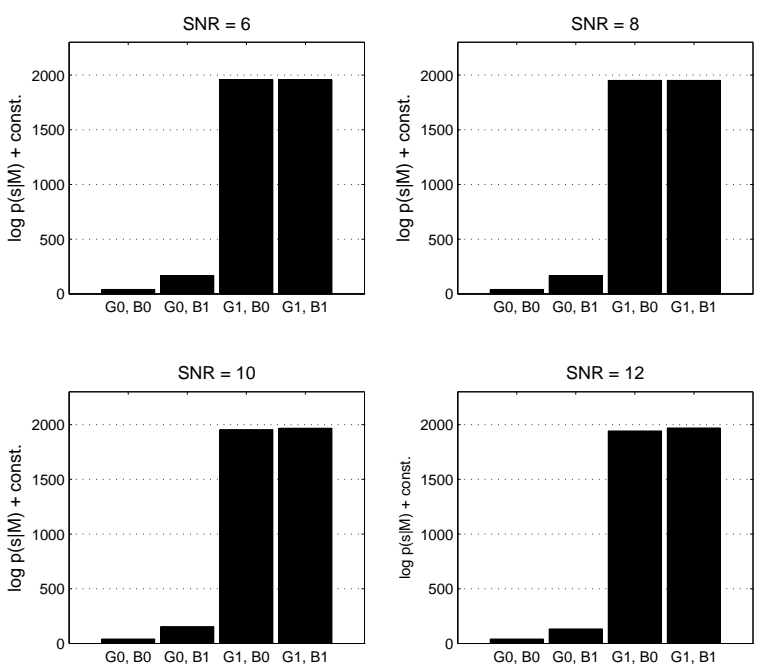

FIG. 9: Log evidence for each model under consideration for Example 1. The evidence is highest for the glitch-fitting models. The $\mathrm{BH}$ plus glitch model $\left[G_{1}, B_{1}\right]$ begins to distinguish itself from the glitch model $\left[G_{1}, B_{0}\right]$ above injected SNR of 8 . Had we naively assumed a Gaussian noise model, the evidence calculation would have erroneously indicated a detection for arbitralily weak signal injections (false positives in the usual parlance).

LIGO/Virgo inspiral search pipeline [3] would not be fooled into claiming a detection from the glitches injected in Examples 1 or 2 (events with significant SNR in just one detector do not make it through to the coincidence test). On the other hand, introducing the capability to model instrument glitches should help lower the thresholds in these searches.

\section{DISCUSSION}

The goal of this work was to develop a better likelihood function (i.e., noise model) to account for non-Gaussian and non-stationary features in LIGO/Virgo data, and to apply this new tool to the gravitational wave detection problem. We studied the performance of three noise models proposed as an alternative to the standard stationary, Gaussian treatment:

1. Noise samples are drawn from a Gaussian distribution with a time-varying expectation value. $\left[N_{0}, G_{0}\right]$

2. The noise distribution is modeled as the sum of two Gaussians such that the bulk of the samples are representative of Gaussian noise but with higher probability for "large sigma" events. $\left[N_{1}, G_{0}\right]$

3. The noise consists of two contributions - non-

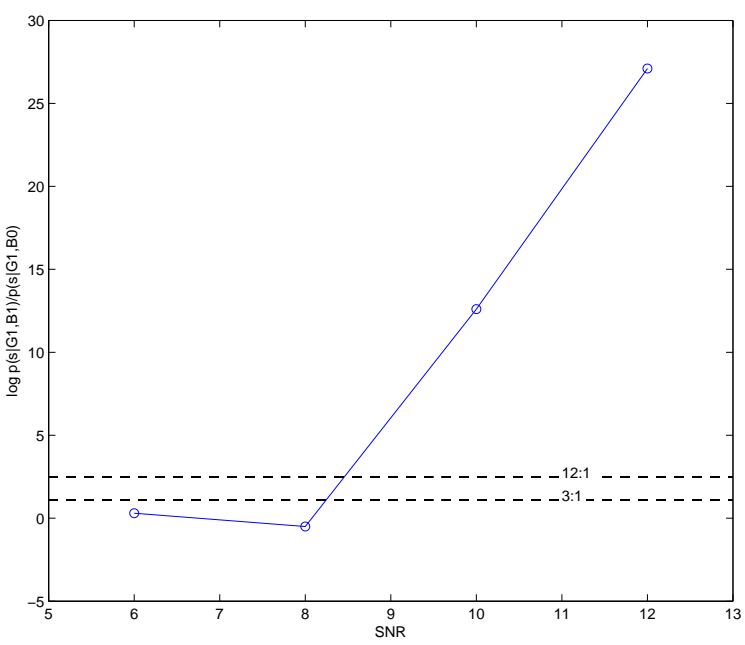

FIG. 10: Log Bayes factor for models $\left[G_{1}, B_{1}\right]$ vs. $\left[G_{1}, B_{0}\right]$ for Example 1. The GW-signal model is favored when the injected signal has SNR above $\sim 8$.

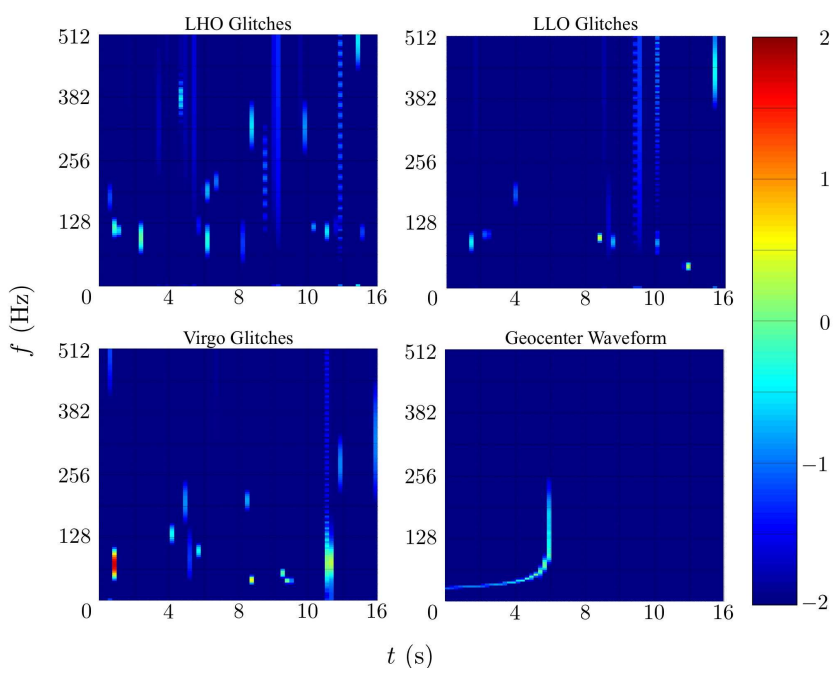

FIG. 11: Spectrograms showing the glitch injections into the three detectors and the geocenter gravitational waveform. The spectrograms have been whitened using the theoretical LIGO/Virgo noise spectra. The color map is on a logarithmic scale, and represents the Fourier power.

stationary, Gaussian samples plus coherent (in time and frequency) "glitches" which are modeled by linear combinations of wavelets. $\left[N_{0}, G_{1}\right]$,

and measured their relative success at modeling different non-Gaussian noise simulations by comparing their marginalized likelihoods.

The most successful of these, $\left[N_{0}, G_{1}\right]$, used a a transdimensional Markov chain Monte Carlo algorithm to determine the most parsimonious number of wavelets used 


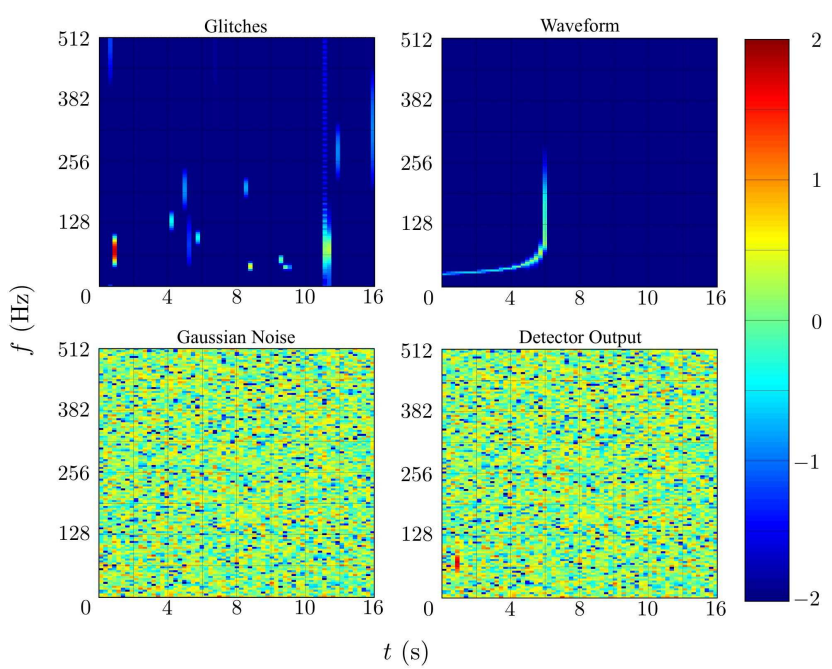

FIG. 12: Spectrograms showing the various components that make up the simulated data in the Virgo detector for Example 2. The spectrograms have been whitened using the theoretical Virgo noise spectrum. The loud, low frequency glitch at $t \sim 1$ second caused havoc for noise models that did not include glitch fitting. The color map is on a logarithmic scale, and represents the Fourier power.

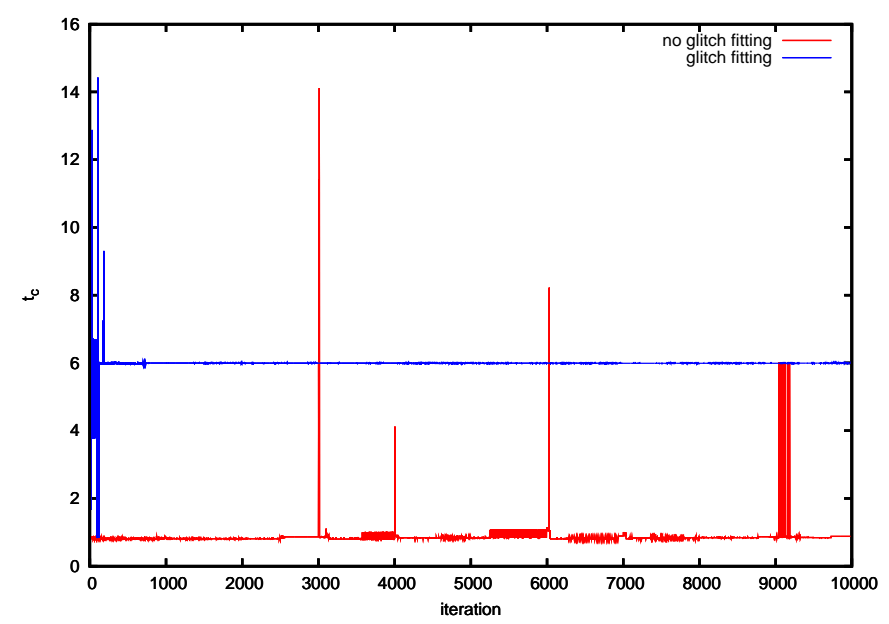

FIG. 13: Time-to-coalescence search chains with and without glitch fitting for Example 2. Without glitch

fitting the search locks onto a glitch in the Virgo detector (injection time $\sim 1 \mathrm{~s}$ ) while with glitch fitting the search locks onto the signal injection $\left(t_{c} \sim 6 \mathrm{~s}\right)$. The

GW signal was injected with SNR of 12 . The prescription for the glitch fitting is described in \$VII.

to fit the non-Gaussian constituents of the noise. This noise model performed as well or better than the others tested for each of the different noise simulations studied (see Figure 4). None of these simulations used either of the noise models to generate the noise samples.
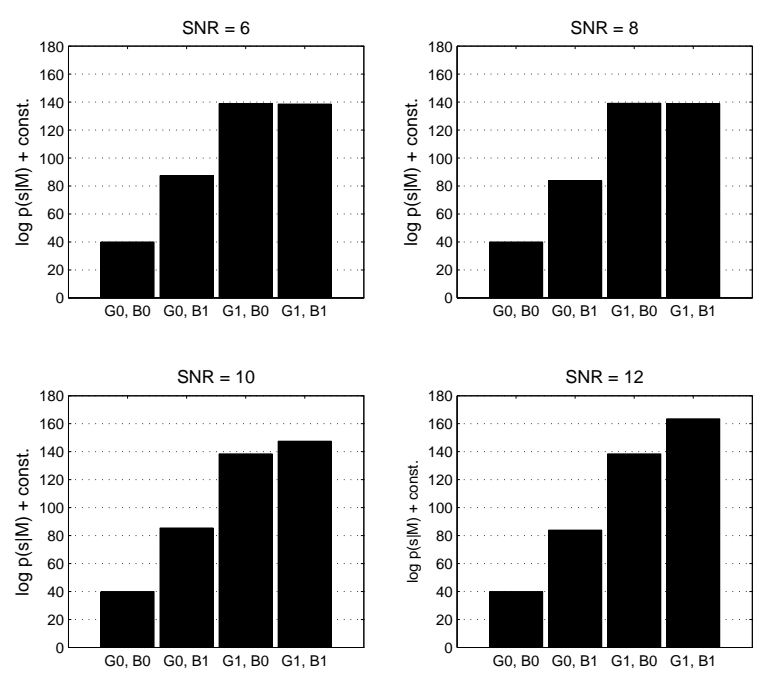

FIG. 14: Log evidence for each model under consideration for Example 2. The evidence is highest for the glitch-fitting models. The $\mathrm{BH}$ model plus glitch model $\left[G_{1}, B_{1}\right]$ is favored for injected SNR's above 8 .

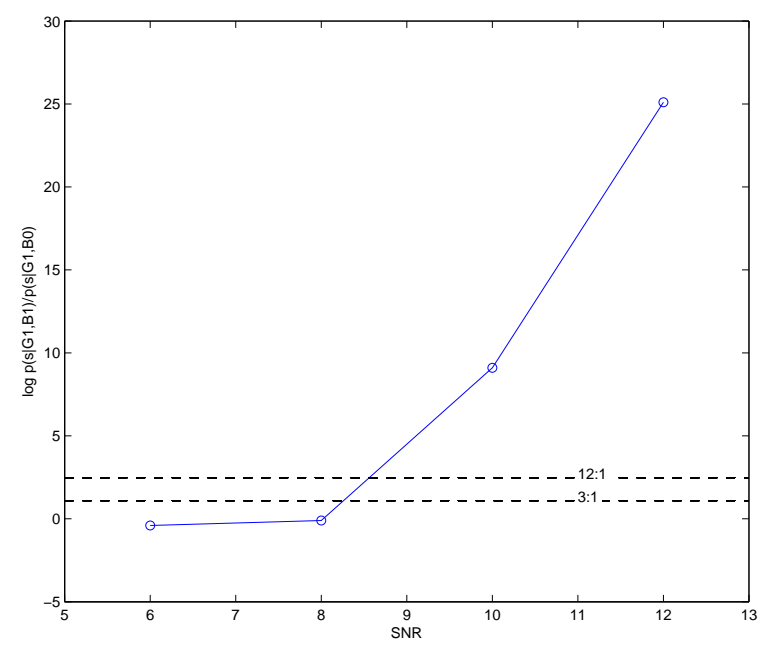

FIG. 15: Log Bayes factor for models with glitch fitting, showing the odds that a signal is present, $\left[G_{1}, B_{1}\right]$, relative to no signal being present, $\left[G_{1}, B_{0}\right]$. The signal model is favored for injected SNR's above 8 .

The more interesting application of the noise modeling is to use it in conjunction with a gravitational wave search. We included the glitch-fitting (along with other improvements) into our parallel tempered Markov chain Monte Carlo detection algorithm originally described in [23]. The study was performed using two simulations of the interferometer data. One contained Gaussian noise plus a single "loud" glitch intentionally overlapping the injected GW. The other contained Gaussian noise with 


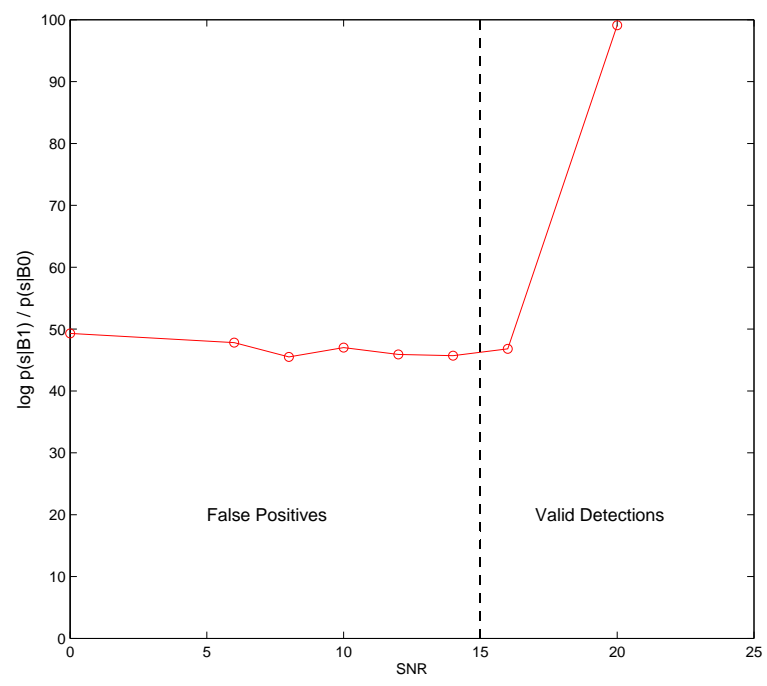

FIG. 16: Log Bayes factor for models without glitch fitting, showing the odds that a signal is present, $\left[G_{0}, B_{1}\right]$, relative to no signal being present, $\left[G_{0}, B_{0}\right]$. The signal model is always favored, even when no signal is injected. The parameters of the recovered signal do not correspond to those injected until the SNR reaches $\sim 16$.

100 glitches distributed across the network having SNRs drawn from a distribution calibrated to match that of single detector's triggers in a LIGO S5 black hole ringdown search. We find no false positive detections when the glitch modeling is included, and are able to locate the gravitational wave signal injected into data with SNR above $\sim 8$ despite its overlapping with injected glitches. Detections are claimed using Bayesian evidence ratios calculated via thermodynamic integration (see Figs 9,10, [14, and 15).

When the glitch fitting is omitted in favor of the nonstationary Gaussian noise model $\left(N_{0}, G_{0}\right)$ the black hole search misses the astrophysical signals and instead attempts to fit to the injected glitches (see Figs. 7 and 13) until the injected SNR exceeds $\sim 15$. These false positive scenarios ubiquitously returned very large Bayes factors in favor of detection. By using a more realistic model for the noise we have mitigated the risk of falsepositive detections due to non-Gaussian features and successfully extracted GW signals without throwing away the "glitchy" data.

We are encouraged by these demonstrated results and are optimistic that this technique can be used as a foundation for modeling the instrument noise in real data. There is, however, still much work to be done. We will proceed down two avenues to improve the utility of our glitch-fitting detection algorithm.

The first step is to study the performance of these models on actual LIGO/Virgo data in an effort to further improve the glitch modeling. In particular, the priors we have constructed for the glitch parameters (including the number, location in time-frequency space, and amplitudes of the glitches) were uninformed by the ongoing LIGO/Virgo detector characterization studies. Folding in this information should improve the effectiveness of the glitch modeling.

There is no guarantee that wavelets are the best functions with which to decompose the glitches - they were chosen in part for their convenience, as they form an orthogonal basis with a diagonal noise correlation matrix for Gaussian noise. We found that large glitches required $\sim 25$ wavelet basis functions, whereas the analyses performed by Principe and Pinto [15, 16] were more efficient at matching the features in the data. Adopting a more parsimonious basis for fitting the glitches, such as wavelet wavepackets [53], could allow the algorithm to dig deeper into the instrument noise and further lower the detection thresholds.

A weakness of our current implementation is the sequential nature of the initial search phase, which starts with glitch modeling and is then followed by a search for inspiral signals in the cleaned data (the characterization phase, by contrast, simultaneously updates the noise and signal models). This sequential approach is safe so long as the gravitational wave signals are everywhere below the instrument noise level, but it would be desirable to perform the glitch removal in way that will not remove loud gravitational wave signals. To this end, we are currently developing a new version of the algorithm that combines the glitch modeling with a search for un-modeled gravitational wave signals. Wavelets are used to represent the two gravitational wave polarizations $h_{+}$and $h_{\times}$at the Geocenter, and these signals are projected onto the detector network with appropriate time delays and amplitudes corresponding to the proposed sky location. It is possible to specify if the signals are un-polarized, or have a particular waveform polarization (circular, elliptical, linear). If a loud gravitational wave signal is present in the data, it is more parsimonious for the signal to be assigned to the Geocenter signal wavelets than the individual instrument wavelets (for the un-polarized search three detectors are required for this to work, while just two detectors are needed for the polarized search). The new algorithm runs very quickly since the signals do not have to be wavelet transformed at each iteration (the timeshifts are done directly in the wavelet domain). While designed to run as a burst search, the algorithm could be used for data conditioning prior to other searches by subtracting the best fit glitch model from each detector and leaving behind any power assigned to the signal model.

\section{ACKNOWLEDGMENTS}

We are grateful for input from Bruce Allen, Paul Baker, Kipp Cannon, Jolien Creighton, Joe Romano and Graham Woan. This work was supported by NSF grant 
0855407.

\section{Appendix: A Recipe for Parallel Tempering and Thermodynamic Integration}

Parallel tempering is an effective way to keep Markov chains from locking onto single mode of the target posterior distribution function, with the added benefit of a simple and accurate means of calculating the model's evidence (thermodynamic integration). Parallel tempering involves running $N_{C}$ chains simultaneously using a modified Hasting's ratio

$$
H_{\vec{\theta}_{x} \rightarrow \vec{\theta}_{y}}=\left(\frac{p\left(s \mid \vec{\theta}_{y}, \beta\right)}{p\left(s \mid \vec{\theta}_{x}, \beta\right)}\right)^{\beta} \frac{p\left(\vec{\theta}_{y}\right) q\left(\vec{\theta}_{x} \mid \vec{\theta}_{y}\right)}{p\left(\vec{\theta}_{x}\right) q\left(\vec{\theta}_{y} \mid \vec{\theta}_{x}\right)}
$$

where $\beta=1 / T$ is analogous to an inverse "temperature" and takes on an increasing value between 0 and 1 for each chain. The effect of the exponent on the likelihood ratio in Eq. 27 is to smooth the topography of the target distribution, making differences between modes of a distribution less dramatic, and broadening the basin of attraction for each mode. A chain with a high temperature (low $\beta$ ) will freely move between modes and in the limit where $T \rightarrow \infty(\beta \rightarrow 0)$ the likelihood ratio goes to 1 and the chain will sample the prior distribution $(p(\vec{\theta}))$.

Hotter chains efficiently sample the full prior volume and, in the event that they locate a region of higher posterior weight, are able to communicate that location to the colder chains without violating detailed balance by using the "chain swapping" Hasting's ratio:

$$
H_{i \leftrightarrow j}=\frac{p\left(s \mid \vec{\theta}_{i}, \beta_{j}\right) p\left(s \mid \vec{\theta}_{j}, \beta_{i}\right)}{p\left(s \mid \vec{\theta}_{i}, \beta_{i}\right) p\left(s \mid \vec{\theta}_{j}, \beta_{j}\right)} .
$$

for an exchange between chains $i$ and $j$. Meanwhile, the colder chains are more apt to thoroughly explore the space around whichever mode it is they sit. It is as if the hot chains wildly try different solutions, and pass those that offer good fits to the data to the cold chains where they can stored in the history of the chain, and refined with subsequent iterations.

Direct exchange of states between two chains is the simplest way to couple the chains, but not the most efficient. The use of genetic algorithms (GAs) to produce new solutions (the offspring) from parallel chains (the parents) further increases the benefits of parallel tempering.

The post-burn-in samples from all of the parallel chains can be used to calculate the evidence for the model under consideration by integrating the average log-likelihood for each chain over $\beta$

$$
\begin{aligned}
\log p(s \mid \mathcal{M}) & =\int_{0}^{1}\langle\log p(s \mid \vec{\theta}, \mathcal{M}, \beta)\rangle d \beta \\
& =\int_{-\infty}^{0} \beta\langle\log p(s \mid \vec{\theta}, \mathcal{M}, \beta)\rangle d \log \beta
\end{aligned}
$$

By repeating the calculation for different models under consideration, one can calculate the Bayes factor (the ratio of the evidence for two models) or, for a larger number of models, the likelihood distribution (in "model space"). If the priors on competing models are uniform, the likelihood distribution is the model posterior up to a normalization constant.

\section{Heating schemes}

An efficient implementation of parallel tempering requires the temperature spacing between chains to be large enough that each is free to find it's own stationary state, but small enough that the chains are still frequently exchanging parameters with one another. At low temperature we tend to err on the side of communication, ensuring that the cold chains are efficiently sampling from modes of the posterior. This could, depending on the model, mandate very close spacing of the chains. On the other hand, as the temperature increases the likelihood ratio becomes increasingly less important in the Hasting's ratio, and parameter exchanges become frequently accepted. In this scenario the chains become over-coupled, preventing them from locating a stationary solution. This, in turn, affects the accuracy of the evidence integration. For Gaussian likelihoods, geometric spacing of chain temperatures is most effective.

We use two temperature spacings, $\delta T_{h}$ and $\delta T_{c}$, for the hotter and colder chains. The change-over between the $\delta T$ s occurrs at some pre-set temperature $T_{*}$. We have typically found that $\delta T_{c} \sim 1.2-1.5$ for the $N_{*}$ (colder) chains yields adequate mixing. A more sophisticated choice would be to adjust $\delta T_{c}$ during the burn-in phase until achieving a desired acceptance rate for the PT proposals.

The temperature spacing for the remaining $N_{C}-N_{*}$ (hotter) chains is calculated such that the hottest chain has temperature $T_{\max }$ with typical values between $10^{2}$ and $10^{4}$. We elect to fix the highest temperature, instead of the spacing for the hotter chains, so that the evidence integrals for different models occur over the same range regardless of which $\delta T_{c}$ or $T_{i}$ we choose for each model.

To determine $T_{*}$ we utilize the approximation that (for Gaussian posteriors) the effective SNR of the signal seen by a tempered chain chain is $\mathrm{SNR}_{\text {eff }} \sim \mathrm{SNR} / \sqrt{\mathrm{T}}$. Because a GW with SNR greater than $\sim 10$ will not generally require careful model-selection (assuming we have done an adequate job of modeling the noise) we have used $T_{*} \sim 10$. This corresponds to a maximum effective SNR at $T_{*}$ for marginally detectable signals of $10 / \sqrt{10} \sim 3$ which is sufficiently small to guarantee that hotter chains will not "see" the GW signal. With the $\delta T$ s determined, the temperature for each chain in the ladder is

$$
\begin{aligned}
& T^{i}=\delta T_{c}^{i}, \quad 0 \leq i<N_{*} \\
& T^{i}=T_{*} \delta T_{h}^{i-N_{*}}, \quad N_{*} \leq i<N_{C} .
\end{aligned}
$$


If locating and/or characterizing the modes of the posterior, without calculating the evidence, is the goal of the analysis the temperature ladder need not extend beyond $N_{*}$. Hotter chains are primarily sampling from the priors and do not noticeably aid in the convergence of the colder chains (although they are critical in the evidence calculation). In other words, chains that are to be used for thermodynamic integration have to go out to much larger temperature than those used to produce samples the posterior. The exact number of chains, and the maximum temperature needed for both scenarios, are tuned on a problem-by-problem basis. Key diagnostics for determining these settings are discussed later.

\section{Proposal distributions}

There are no rules mandating how often, or between which chains, parameter exchanges should be proposed. We have typically proposed exchanges only between adjacent chains, and done so between each chain once for every iteration of the MCMC. The parameter-swap proposal will first suggest an exchange between chains $N_{C} \leftrightarrow N_{C}-1$ (the hottest, and next to hottest chains), then $N_{C}-1 \leftrightarrow N_{C}-2$, and so on, until a proposed parameter switch between chains $1 \leftrightarrow 0$ (the next to coldest and coldest chains). For model selection problems, when the maximum temperature is large, the hotter chains (each effectively sampling from the the prior) accept chainswaps with excessively high frequency ( $>90 \%)$. To prevent the hotter chains from over-coupling with their neighbors we propose exchanges between chains $i$ and $j$, where $T_{j}<T_{i}$ with probability $\beta_{j}$. The coldest chain has $\beta=1$ and always attempts parameter exchanges during the PTMCMC proposal while hotter chains attempt to exchange parameters more rarely

Proposal distributions within the chains are also informed by the chain temperature. Hotter chains should attempt large jumps in parameter space so they rapidly explore the entire prior volume, while colder chains should take smaller jumps so they efficiently accept new states in the chain. It is natural to scale the jumps by the temperature

$$
\delta \theta \rightarrow \delta \theta \sqrt{T_{i}}
$$

which is, again, motivated by the assumption that the target distribution is Gaussian. For the hotter chains, this assumption is no longer valid, and these jumps can become large enough that they greatly exceed the prior ranges, or are never accepted if the priors are informative (i.e., have regions of high probability). Some care needs to be taken when scaling proposals by the chain temperature to avoid quenching the free-range exploration of the hottest chains. The hotter chains can frequently encounter the bounds of the prior volume. This can enhance the need to carefully deal with jumps that are outside of the prior range (for instance, using jumps along eigenvectors of the Fisher matrix when the current solution is near the edge of the prior). We have used periodic and reflecting boundary conditions on the prior volume. Simply rejecting proposals that are out of bounds violates the detailed balance condition.

\section{Diagnostics}

In our experience, the most important diagnostic tool to use when testing an MCMC algorithm is to verify that the target distribution of a $T=\infty$ chain is identical to the prior distribution. Because you can do this study without ever calculating the likelihood (often the most computationally demanding part of each iteration) this is a quick, invaluable test.

It is also important to note that burn-in times for hot and cold chains can be very different depending on the proposal distributions and the size of the prior ranges for the model parameters. It is always a good idea, at least during the testing of the algorithm, to store each temperatures chain.

Other useful figures of merit are plots of $\langle\log p(s \mid \vec{\theta}, \mathcal{M}, \beta)\rangle$ versus $\beta$. Figures 17 and 18 are annotated with some of the more valuable diagnostic features. In these examples, $M_{1}$ has a higher dimension than $M_{0}$ and was the more appropriate model. The higher dimensional model should normally have equal or (more often) higher likelihood at low temperature than a model with fewer degrees of freedom. Exceptions to this rule are possible if the two models are not nested (i.e., one model is not a limiting case of the other). We have exclusively used these methods to distinguish between nested models (most often the GW detection problem) so the following discussion assumes that to be the case.

The average log-likelihood should increase smoothly and monotonically with increasing $\beta$. A jagged $\langle p(s \mid \vec{\theta})\rangle$ curve is indicative of large errors in the calculation of the average log-likelihood, typically associated with poor convergence of the chains. Longer run times, more efficient proposal distributions, or different heating schemes can all help improve the convergence rates thus reducing the variance of the likelihood chain.

At low temperature, $M_{1}$ is able to achieve a better fit to the data and therefore returns a higher likelihood. This is true even if $M_{1}$ is not the best description of the data due to its additional degrees of freedom. The high temperature chains supply the "Occam factor," or penalty for carrying that additional flexibility.

The point at which $M_{0} \simeq M_{1}$ (the "equilibrium temperature") indicates when the high-dimensional model chain has become sufficiently hot that the "extra" model parameters lose touch with whatever feature they had been fitting, and begin predominantly sampling the priors (see figure 17). At this point it is safe to switch to the larger chain spacing, however the location of $T_{*}$ isn't something known a priori. Some educated tuning/guessing is needed to determine the location of such a 


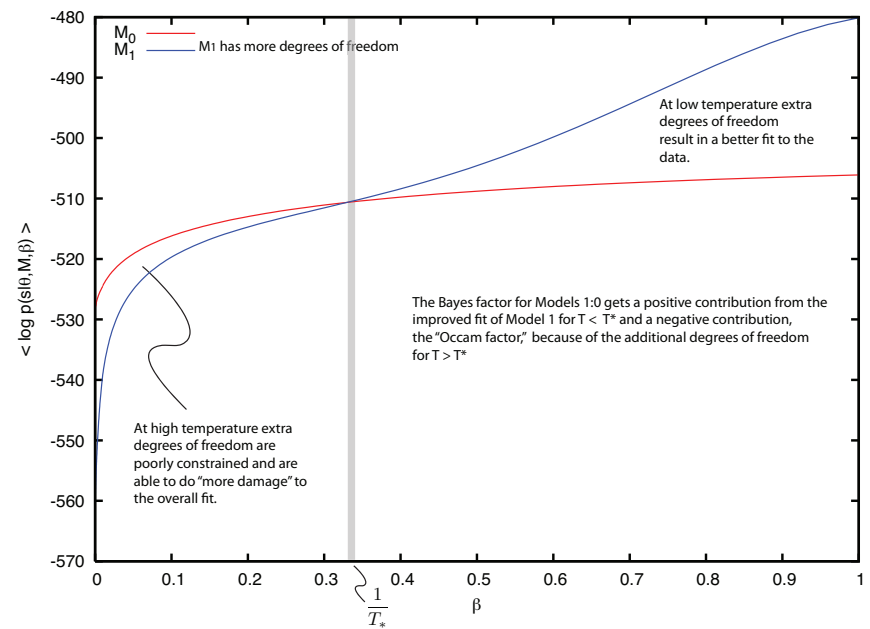

FIG. 17: An example $\langle\log \mathcal{L}\rangle$ vs. $\beta$ plot showing the location of $T_{*}$ and other tips to understanding how parallel tempering and thermodynamic integration work. In this example the blue curve is for the model with a higher number of degrees of freedom, and was the favored explanation of the data.

transition point. We can validate the choice of $T_{*}$ by ensuring that it is lower than the equilibrium temperature of the chains.

The temperature range where a model switches from fitting to the data and sampling from the priors can result in a steep drop in average likelihood, especially for high dimensional models. This large transition can be difficult to sample with chains automatically, as it can occur after the temperature spacing has been increased. If it is poorly resolved, the exact interval between chains where change occurs is not convergent between trial runs. Should this be the case, an additional block of finely sampled (in temperature) chains should be inserted around that region.

At high temperature, the model with more flexibility is no longer constrained by the data, and the extra degrees of freedom result in, on average, a much worse fit to the data.

To clarify this, consider the case where the the two models under consideration are that of the detection problem (for this discussion we will assume stationary Gaussian noise and uninformative priors). A noise-only model will not be able to accommodate a GW signal in the data, while a template will fit to the GW signal (if there is one) or to some part of the noise (if there is not). Regardless of the data, $M_{1}$ will return a higher likelihood than $M_{0}$ for low temperature. At high tem- perature, $M_{1}$ is sampling from the priors on the GW parameters without being significantly informed by the data because the likelihood ratio in the transition probability is suppressed by the temperature. Therefore, high temperature $M_{1}$ chains are likely to include in the data a bright GW signal which can result in a large $\chi^{2}$ and accordingly, a low value for the likelihood. Colloquially, $M_{1}$

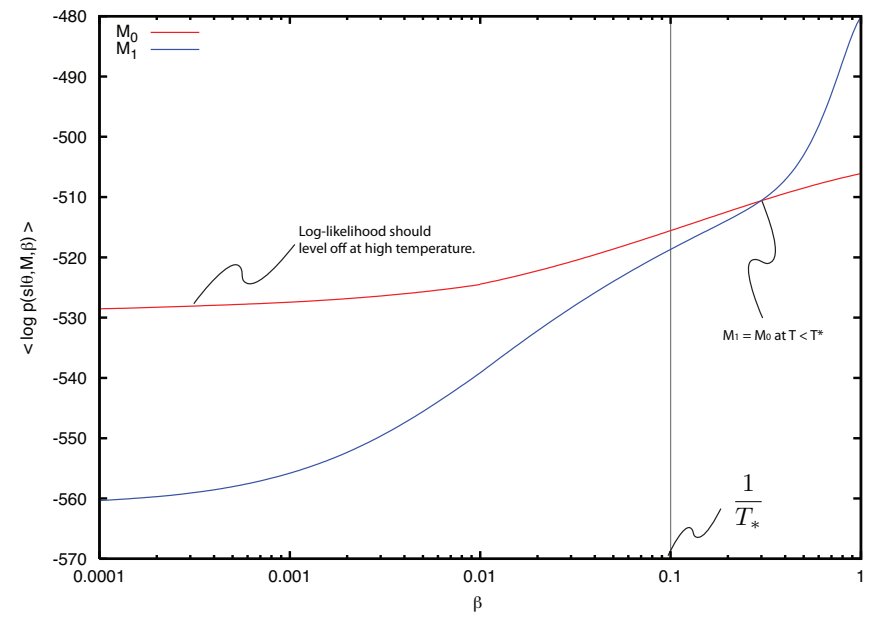

FIG. 18: The same plot as is found in figure 17 but on a $\log$ scale in $\beta$. The log-version of these plots is a valuable diagnostic for the behavior of the chains at high temperature. This also shows the "rule of thumb" value for $T_{*}$ which passes the test of being lower than the point where $M_{1}=M_{0}$.

can do a lot of "damage" to the residual if it is effectively unconstrained.

For thermodynamic integration, the model selection question comes down to whether or not the improvement in likelihood for the colder chains can overwhelm the Occam factor supplied by the hotter chains. This emphasizes the importance of choosing an appropriate $T_{\max }$. The average likelihood stops decreasing once the temperature is high enough that additional chains in the ladder produce samples from identical distributions (the prior). This shows up as a floor in the likelihood versus inverse temperature plot best seen in Fig 18. Beyond this point, contributions to the evidence integral are proportional to $\beta$ (which is approaching zero) and can safely be neglected.
[1] A. Abramovici et al., Science 256, 325 (1992).

[2] http://www.virgo.infn.it/

[3] B. P. Abbott et al. [LIGO Scientific Collaboration], Phys.
Rev. D 79, 122001 (2009) arXiv:0901.0302 [gr-qc]].

[4] B. P. Abbott et al., Phys. Rev. D80, 102001 (2009).

[5] P. Laplace Essai philosophique sur les probabilits. (1814). 
[6] M. V. van der Sluys, C. Roever, A. Stroeer, N. Christensen, V. Kalogera, R. Meyer and A. Vecchio, arXiv:0710.1897 [astro-ph].

[7] M. van der Sluys et al., Class. Quant. Grav. 25, 184011 (2008) arXiv:0805.1689 [gr-qc]].

[8] M. van der Sluys, I. Mandel, V. Raymond, V. Kalogera, C. Rover and N. Christensen, Class. Quant. Grav. 26, 204010 (2009) arXiv:0905.1323 [gr-qc]].

[9] B. Allen, J. D. E. Creighton, E. E. Flanagan, and J. D. Romano, Phys. Rev. D67, 122002 (2003).

[10] B. Allen, J. D. E. Creighton, E. E. Flanagan, and J. D. Romano, Phys. Rev. D65, 122002 (2002).

[11] J. Clark, I. S. Heng, M. Pitkin and G. Woan, Phys. Rev. D 76, 043003 (2007) arXiv:gr-qc/0703138.

[12] J. Veitch and A. Vecchio, Phys. Rev. D 81, 062003 (2010) arXiv:0911.3820 [astro-ph.CO]].

[13] P. Ajith, M. Hewitson, J. R. Smith, H. Grote, S. Hild and K. A. Strain, Phys. Rev. D 76, 042004 (2007) arXiv:0705.1111 [gr-qc]].

[14] P. Ajith, R. Adhikari and A. Pearlman, LIGO Document T0900456-v2 (2009). https://dcc.ligo.org/DocDB/0005/ T0900456/002/FinalReport_v2.pdf. See also slides 10 \& 11 of https://www.lsc-group.phys.uwm.edu/twiki/pub /DetChar/NOV2009/InsVetoes_Ajith.pdf

[15] M. Principe and I. M. Pinto, Class. Quant. Grav. 25, 075013 (2008) arXiv:0806.4574 [gr-qc]].

[16] M. Principe and I. M. Pinto, Class. Quant. Grav. 26, 045003 (2009).

[17] K. C. Cannon, Class. Quant. Grav. 25, 105024 (2008).

[18] K. S. Thorne, in Gravitational Radiation in 300 Years of Gravitation, edited by S. W. Hawking and W. Israel (Cambridge University Press, ADDRESS, 1987), No. pp 330-458.

[19] C. Cutler and M. Vallisneri, Phys. Rev. D76, 104018 (2007).

[20] L. S. Finn, Phys. Rev. D46, 5236 (1992).

[21] N. J. Cornish and T. B. Littenberg, Phys. Rev. D76, 083006 (2007).

[22] M. R. Adams and N. J. Cornish, arXiv:1002.1291 [gr-qc] (2010).

[23] T. B. Littenberg and N. J. Cornish, Phys. Rev. D80, 063007 (2009).

[24] S. Klimenko, I. Yakushin, A. Mercer, and G. Mitselmakher, Class. Quant. Grav. 25, 114029 (2008).

[25] W. Press, S. Teukolsky, W. Vetterling, and B. Flannery, Numerical Recipes in C, The art of Scientific Computing, 2 ed. (Cambridge University Press, ADDRESS, 1993).

[26] N. Metropolis, A. Rosenbluth, R. M.N., A. Teller, and E. Teller, J. Chem. Phys. 21, 1087 (1953).

[27] W. Hastings, Biometrika 57, 97 (1970).

[28] J. Skilling and D. MacKay, BayeSys/Nested sampling.

[29] N. Christensen and R. Meyer, Phys. Rev. D 58, 082001
(1998)

[30] N. Christensen and R. Meyer, Phys. Rev. D 64, 022001 (2001) arXiv:gr-qc/0102018.

[31] N. J. Cornish and J. Crowder, Phys. Rev. D72, 043005 (2005).

[32] J. Crowder and N. Cornish, Phys. Rev. D75, 043008 (2007).

[33] N. J. Cornish and E. K. Porter, Class. Quant. Grav. 24, S501 (2007).

[34] J. Gair, E. K. Porter, S. Babak, and L. Barack, Class. Quant. Grav. 25, (2008).

[35] D. A. Brown, J. Crowder, C. Cutler, I. Mandel, and M. Vallisneri, Class. Quant. Grav. 24, S595 (2007).

[36] E. L. Robinson, J. D. Romano, and A. Vecchio, Class. Quant. Grav. 25, 184019 (2008).

[37] M. Trias, A. Vecchio, and J. Veitch, Class. Quant. Grav. 25, 184028 (2008).

[38] J. S. Key and N. J. Cornish, Phys. Rev. D79, 043014 (2009).

[39] R. Swendsen and J. Wang, Phys. Rev. Lett. 57, 2607 (1986).

[40] P. Goggans and Y. Chi, in Bayesian Inference and Methods in Science and Engineering, edited by J. Rychert, G. Erickson, and C. Smith (American Institute of Physics, USA, 2004).

[41] F. Feroz and M. P. Hobson, arXiv:0704.3704 [gr-qc] (2007).

[42] F. Feroz, J. R. Gair, M. P. Hobson, and E. K. Porter, arXiv:0904.1544 [gr-qc] (2009).

[43] D. Sivia and J. Skilling, Data Analysis: A Bayesian Tutorial (Oxford University Press, Great Clarendon Street, Oxford OX2 6DP, 2006), No. ISBN 0198568312.

[44] P. Green, in Highly Structured Stochastic Systems, edited by P. Green, N. Hjort, and S. Richardson (Oxford University Press, ADDRESS, 2003).

[45] E. K. Porter and N. J. Cornish, arXiv:0804.0332 [gr-qc] (2008).

[46] A. Buonanno et al., Phys. Rev. D 76, 104049 (2007).

[47] Y. Pan et al., Phys. Rev. D 81, 084041 (2010).

[48] R. N. Lang and S. A. Hughes, arXiv:0710.3795 [gr-qc] (2007).

[49] S. T. McWilliams, J. I. Thorpe, J. G. Baker, and B. J. Kelly, Phys. Rev. D81, 064014 (2010).

[50] J. S. Key and N. J. Cornish, arXiv:1006.3759 [gr-qc] (2010).

[51] J. Veitch and A. Vecchio, Phys. Rev. D 78, 022001 (2008) arXiv:0801.4313 [gr-qc]].

[52] W. Farr In preparation

[53] R. R. Coifman and M. V. Wickerhauser, IEEE Trans. Inf. Theory 38, 713 (1992). 


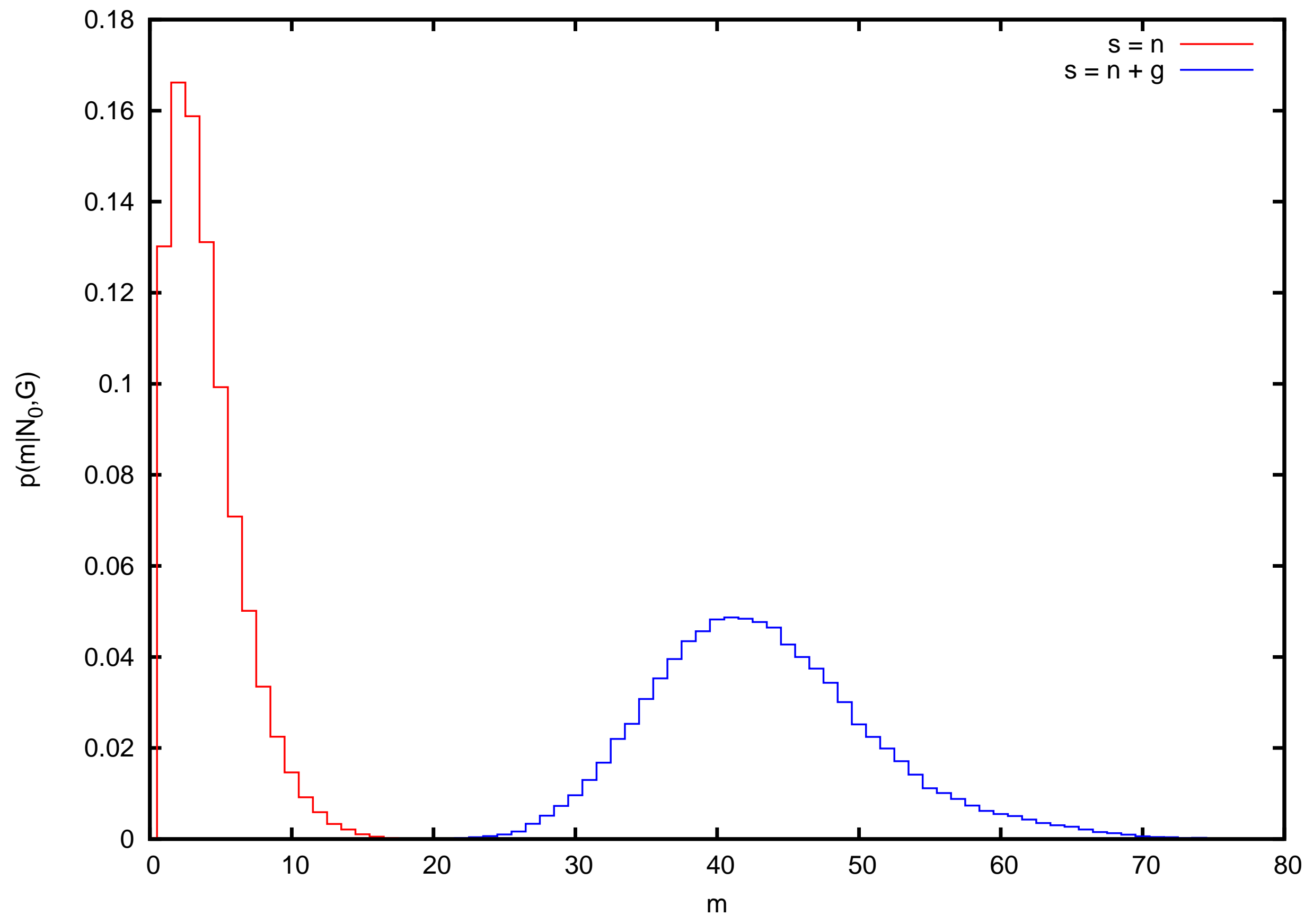

